\title{
Common Features of Core "Electron-Root” Confinement in Helical Devices
}

\author{
M.Yokoyama, H.Maaßberg*, C.D.Beidler*, V.Tribaldos**, K.Ida, F.Castejon**, \\ T.Estrada**, A.Fujisawa, T.Minami, T.Shimozuma, Y.Takeiri, J.Herranz**, S.Murakami*** \\ and H.Yamada
}

National Institute for Fusion Science, Toki 509-5292, Japan

Max-Planck Institut fur Plasmaphysik, Greifswald 17491, Germany*

Laboratorio Nacional de Fusion, CIEMAT-FUSION, Madrid 28040, Spain**

Department of Nuclear Engineering, Kyoto Univ., Kyoto 606-8501, Japan***

Information on the corresponding author

Masayuki YOKOYAMA

National Institute for Fusion Science,

322-6 Oroshi, Toki 509-5292, Japan

yokoyama@nifs.ac.jp

FAX: $+81-572-58-2630$

Total number of

Pages : 34 (including References, Figure Captions)

Figures : 8 


\begin{abstract}
The characteristics of core electron-root confinement (CERC) in helical devices are illustrated using results from the four different experiments, CHS, LHD, TJ-II and W7-AS. Common features include strongly peaked electron temperature profiles and large positive radial electric fields, $E_{\mathrm{r}}$, in the core region for discharges with sufficient central electron cyclotron heating $(\mathrm{ECH})$. Such observations are consistent with a transition to the "electron-root" solution of the ambipolarity condition for $E_{\mathrm{r}}$, a feature of neoclassical theory which is unique to non-axisymmetric configurations. The magnetic topology of the configuration plays a role in this transition and thresholds are found for the particle density and ECH power, in accordance with neoclassical expectations. Neoclassical theory alone cannot explain all observations, however, as CERC formation can also be influenced by ECH-driven convective fluxes of localized electrons and by the presence of magnetic islands in the core region. This is the first report describing collaborative activities within the framework of the International Stellarator Profile Data Base.
\end{abstract}




\section{Introduction}

Core "electron-root" confinement (CERC) is an improved confinement regime which is stellarator-specific, i.e. it does not exist in tokamaks. It is characterised by highly peaked electron temperature profiles (core confinement) together with large (greater than a few $\mathrm{kV} / \mathrm{m}$ ) positive radial electric fields (electron-root). This regime has been obtained in quite different helical devices for electron cyclotron heated $(\mathrm{ECH})$ discharges above a power threshold depending on the specific magnetic configuration.

The transition to improved "electron-root" confinement is different than the formation of an internal transport barrier (ITB) observed in tokamaks where the strongly sheared $\mathbf{E} \times \mathbf{B}$ flow within the narrow barrier screens off the anomalous transport driven by ITG and TEM turbulence (ETG, being a small-scale turbulence, is not affected by this $\mathbf{E} \times \mathbf{B}$ flow); see e.g. the reviews [1,2] for more details. In tokamaks, the ITB physics dominates both the particle and the ion energy balance whereas in helical devices CERC affects primarily the electron energy balance. A detailed comparative analysis of CERC and ITB signatures for LHD and JT-60U is given in Ref. [3].

According to neoclassical theory for the stellarator long-mean-free-path $(\operatorname{lm} f p)$ regime, the radial electric field, $E_{\mathrm{r}}$, established in the electron root is of sufficient magnitude to limit the radial displacement of ripple-trapped electrons due to the $\nabla B$-drift, and thereby suppresses $1 / \nu$-regime transport ( $v$ being the collision frequency) which holds for small $E_{\mathrm{r}}[4,5]$. Circumvention of this unfavorable $1 / v$-regime, for which the heat flux density scales with $T_{\mathrm{e}}^{9 / 2}$, is one of the major goals for stellarator optimisation [6]. This is usually treated as a matter of optimising the magnetic configuration, either by reducing the fraction of ripple-trapped particles (concept of quasisymmetry; in particular, the quasi-helically symmetric HSX [7] and the quasi-axisymmetric NCSX [8]) or by drift optimisation (concept of quasi-isodynamicity; see e.g. [9] for W7-X). The 
suppression of $1 / v$ transport via the electron root makes another strategy conceivable and understanding the physics of the CERC results in CHS, LHD, TJ-II and W7-AS and their ramifications for such an "electron-root perspective" is one of the main motivations of this work.

The transitions between the "ion-root" (with small magnitude of $E_{\mathrm{r}}$, usually negative) and the "electron-root" (with large positive $E_{\mathrm{r}}$ ) are based on a bifurcation mechanism if the ambipolarity condition of the neoclassical fluxes allows for several roots (an odd number). This feature leads to thresholds with respect to density and ECH power depending on the magnetic configuration as well as on the position of the ECH deposition (e.g. in $B$-scans). Close to these thresholds, triggered and spontaneous transitions can be found both in the central potential (measured with the heavy ion beam probe, HIBP) and in the central electron temperature profile (obtained from the electron cyclotron emission, ECE); an example of both time traces taken for the same discharge in TJ-II can be found in Ref. [10].

Historically, CERC was observed in CHS and in W7-AS at nearly the same time. From the excellent HIBP diagnostic with high radial resolution at CHS [11], a central peaking of the positive potential, i.e. strongly positive $E_{\mathrm{r}}$, was reported [12], and the bifurcation character of the fast transitions in $E_{\mathrm{r}}$ was identified [13]. Since the shear in the poloidal $\mathbf{E} \times \mathbf{B}$ flow within the narrow transition region also reduced density fluctuations [14], in a manner fairly similar to that of the tokamak ITBs, the expression "neoclassical internal transport barrier" (N-ITB) was used at CHS.

At W7-AS, a peaking of the central $T_{\mathrm{e}}$ was found both from the ECE and the Thomson scattering diagnostics, and the CERC was indicated from the comparison of the experimental heat diffusivity with the neoclassical predictions [15]. The $E_{\mathrm{r}}$ evaluated from the active CXRS (charge exchange recombination spectroscopy) diagnostic supported the hypothesis of the neoclassical "electron-root" [16], and the name "electron-root feature" was therefore introduced. The discharges at LHD with 
very pronounced peaking of the $T_{\mathrm{e}}$-profile [17] (this was called "electron internal transport barrier", e-ITB) as well as at TJ-II (here, the name "enhanced heat confinement", EHC, was introduced [18]) have confirmed the findings of CHS and W7-AS. As a new aspect, the impact of low-order rational values in the rotational transform on the CERC formation has been recognised $[17,19,20]$ which is outside the framework of the local neoclassical theory.

Recently, an activity to establish an international stellarator profile database (ISPDB) has been initiated. An agreement has been obtained to start with the stellarator-specific CERC for which four helical devices, namely CHS, LHD, TJ-II and W7-AS, can supply experimental results. For the first joint paper produced by this collaboration, it was thought appropriate to adopt the new common name "core electron-root confinement" to replace the different old expressions (expressions containing ITB have sometimes led to confusion since they imply a link to tokamak-ITBs which does not exist). As the first result of this international collaboration, the comparison as well as the documentation of the CERC in four quite different helical devices stresses the entirely different physics in stellarators and in tokamaks.

Section 2 summarises briefly those aspects of neoclassical theory which are relevant for the CERC interpretation. In section 3, the four helical devices contributing to this review are described with respect to their quite different magnetic configurations and their typical ECH operation regimes, in particular to the sensitivity of the power deposition depending on the magnetic topology. In addition, the main diagnostics which are important for this documentation are briefly described. Section 4 compares all main features of CERC in the helical devices and summarises the common signatures. Finally, Section 5 gives the conclusions of this inter-machine analysis as well as an outlook for future optimised stellarator configurations, in particular for NCSX and W7-X. 


\section{Theoretical Background}

Heliacs, heliotrons, stellarators and torsatrons (referred to here collectively as helical devices) produce their confining magnetic fields using different arrangements of current-carrying coils, situated entirely outside of the plasma confinement volume. Although this has obvious advantages (steady-state, disruption-free operation), the necessarily three-dimensional nature of such magnetic fields generally implies a significant fraction of localized particles with large neoclassical transport rates (relative to axisymmetric tokamaks) at fusion-relevant plasma parameters.

The neoclassical theory referred to here takes the linearized drift-kinetic equation as its starting point, assuming that the solution for the distribution function can be accurately described as the sum of a local Maxwellian, $f_{\mathrm{m}}$, and the small departure there from, $f_{1}$. This is equivalent to assuming that the transport across magnetic flux surfaces is very small compared to the transport within the flux surfaces. As a consequence, the flux-surface label, $r$ (the effective minor radius is used here), and the kinetic energy, $\kappa$, appear only as parameters (not as variables) in the equation to be solved for $f_{1}$, and this equation is therefore referred to as both local and mono-energetic. Assuming solutions for $f_{1}$ can be determined, all radial neoclassical transport coefficients can be calculated from the single mono-energetic quantity, $D$, the so-called mono-energetic diffusion coefficient. For example, the radial particle and heat flux densities, $\Gamma_{\alpha}$ and $Q_{\alpha}$, respectively, are given by

$$
\begin{aligned}
& \Gamma_{\alpha}=-D_{1}^{\alpha} n_{\alpha}\left\{\frac{n_{\alpha}^{\prime}}{n_{\alpha}}-\frac{q_{\alpha} E_{\mathrm{r}}}{T_{\alpha}}+\left(\frac{D_{2}^{\alpha}}{D_{1}^{\alpha}}-\frac{3}{2}\right) \frac{T_{\alpha}^{\prime}}{T_{\alpha}}\right\} \\
& Q_{\alpha}=-D_{2}^{\alpha} n_{\alpha} T_{\alpha}\left\{\frac{n_{\alpha}^{\prime}}{n_{\alpha}}-\frac{q_{\alpha} E_{\mathrm{r}}}{T_{\alpha}}+\left(\frac{D_{3}^{\alpha}}{D_{2}^{\alpha}}-\frac{3}{2}\right) \frac{T_{\alpha}^{\prime}}{T_{\alpha}}\right\}
\end{aligned}
$$


where $\alpha$ is the species index ( $\alpha=$ e for electrons, $\alpha=\mathrm{i}$ for ions), $n$ is the density, $q$ the charge, $T$ the temperature, $E_{\mathrm{r}}$ the radial electric field and a prime denotes differentiation with respect to $r$. The transport coefficients are determined from

$D_{j}^{\alpha}=\frac{2}{\sqrt{\pi}} \int_{0}^{\infty} d x_{\alpha} x_{\alpha}^{(2 j-1) / 2} D\left(x_{\alpha}\right) e^{-x_{\alpha}}$

which is a weighted convolution of $D$ with the local Maxwellian, written here in terms of the normalized kinetic energy $x_{\alpha}=\kappa_{\alpha} / T_{\alpha}$.

In the $\operatorname{lmfp}$ in helical devices, localized particles experience a uni-directional radial drift until pitch-angle collisions scatter them out of the local magnetic ripple in which they are trapped. This is the physical explanation for the so-called $1 / v$ regime with less frequent collisions leading to a larger neoclassical transport. The radial excursion of the localized particles is limited, however, by the presence of a radial electric field, the $\mathbf{E} \times \mathbf{B}$ drift causing the localized particles to precess poloidally with frequency $\Omega_{\mathrm{E}}=E_{\mathrm{r}} / r B_{0}$. For the simplest of helical devices, the transport now scales either as $\sqrt{v}$ or $v$, depending on whether collisions or drifts are responsible for removing the particles from local ripples. It is possible to determine $D$ in each of these three $\operatorname{lm} f p$ regimes from asymptotic solutions of the kinetic equation and the scalings obtained may be summarized as $[4,21]$

$$
D_{1 / v} \propto \frac{\kappa^{2}}{v} \varepsilon_{\mathrm{eff}}^{3 / 2} \quad D_{\sqrt{v}} \propto \frac{\kappa^{2} \sqrt{v}}{\left|\Omega_{\mathrm{E}}\right|^{3 / 2}} \quad D_{v} \propto\left(\frac{\kappa}{\Omega_{\mathrm{E}}}\right)^{2} v
$$

where $\varepsilon_{\text {eff }}$ is the effective ripple for $1 / v$ transport. These results, when combined with the ambipolarity constraint on the radial particle fluxes, $\Gamma_{\mathrm{e}}=\Gamma_{\mathrm{i}}$, lead to a non-linear equation which can have multiple solutions for $E_{\mathrm{r}}$ [22]. This is a feature of $\operatorname{lm} f p$ neoclassical transport theory for helical 
devices which has no counterpart for axisymmetric tokamaks (where the transport coefficients are independent of $E_{\mathrm{r}}$ ).

Several observations can be made at this point which are of relevance to the experimental results presented in Section 4. Assuming that electrons are in the $1 / v$ regime and that $T_{\mathrm{e}} \approx T_{\mathrm{i}}$, the electric field must reduce the ion particle flux to the electron level and the electrons are therefore said to be the rate-controlling species. Often in this case, only a single solution for $E_{\mathrm{r}}$ exists, usually negative, which is referred to as the ion root (solutions of the non-linear ambipolarity condition traditionally being determined using iterative root-finding techniques). To a good approximation $v \propto \kappa^{-3 / 2}$ so that the $1 / v$ transport coefficients scale as $T^{7 / 2}$. Any increase in $T_{\mathrm{e}}$ therefore leads to rapid growth in $\Gamma_{\mathrm{e}}$ (assuming other quantities to be unchanged) which the ions, of course, must follow. This process requires a reduction in the magnitude of $E_{\mathrm{r}}$ since even if $T_{\mathrm{i}}$ increases with $T_{\mathrm{e}}$ the transport coefficients in the $\sqrt{v}$ and $v$ regimes scale only as $T^{5 / 4}$ and $T^{1 / 2}$, respectively. At some point, however, a second solution for $E_{\mathrm{r}}$ becomes possible (a third solution appears simultaneously but is thermodynamically unstable) which is positive and of large magnitude so as to suppress the $1 / v$ transport of electrons. This solution is called the electron root and the ions are now the ratecontrolling species due to their much smaller transport coefficients. The strong reduction of both ion and electron transport coefficients leads to predictions of significantly improved neoclassical confinement when the electron root is realized.

Two other possibilities for increasing electron $1 / v$ losses are to lower the density $(v \propto n)$ and increase the effective ripple. This latter quantity condenses all effects of the magnetic topology on $1 / v$ transport into a single number. Stellarator optimization has usually concentrated on reducing $\varepsilon_{\text {eff }}$, either through quasi-symmetries with a very small fraction of localized particles [7,8] 
or by drift optimization of the magnetic field topology [9]. Reduction of $\varepsilon_{\text {eff }}$ is an optimization for ion-root operation with small $1 / v$ electron losses responsible for good neoclassical confinement of the entire plasma. If operation at lower density and higher temperature proves to be desirable (or necessary), however, electron-root scenarios are more attractive and these are easier to realize with a large $\varepsilon_{\text {eff }}$.

To obtain accurate results for transport coefficients in realistic helical devices, one must abandon simple models for $B$ and employ numerical methods which are capable of accounting for arbitrarily complex magnetic fields [23-26]. These methods have been extensively benchmarked within an international collaboration and exhibit excellent agreement in all cases [27]. For the four helical devices considered here, it was also possible to verify the theoretically predicted scalings of $D$ for each of the three $\operatorname{lmfp}$ regimes, in particular the strong reduction of the mono-energetic diffusion coefficient due to the radial electric field.

To perform the neoclassical analysis of experimental discharges, the measured plasma profiles are combined with pre-computed tables of normalized values of $D$ (depending only on $r, E_{\mathrm{r}} / \mathrm{v} B_{0}$ and $v / \mathrm{v}$, where $\mathrm{v}$ is the velocity) and the value of $E_{\mathrm{r}}$ is varied to find all roots of the ambipolarity condition. For CERC discharges, the strong central peaking of the $T_{\mathrm{e}}$ profile allows only the electron-root solution to appear in the plasma core, while in the periphery with $T_{\mathrm{e}} \approx T_{\mathrm{i}}$ and significant density gradient only the ion root is typically realized (at very low density, an electron root can also be found at the edge; see e.g. [28] for LHD). Between "core" and "periphery" lies, in general, a region in which multiple roots exist and by tracing out, in succession, the radial profiles of electron, unstable and ion roots, one obtains a characteristic " $\mathrm{S}$ " curve of the type familiar from first-order phase transitions in non-linear thermodynamics [29]. Pursuing this thermodynamic approach, the radius at which the bifurcation from electron to ion root occurs is chosen so that the 
generalized heat production rate of the neoclassical particle transport is identical for both roots [30]. Treating $r$ as the bifurcation parameter in this manner must be understood as a convenient means for characterizing all profile and configuration data with a single quantity; where such experiments can be performed, it is equally valid to speak of the density or heating power as relevant for the bifurcation.

Before leaving this section, it should be emphasized that the neoclassical theory used here is based on the assumption of local transport (assuming closed, nested flux surfaces) and is therefore incapable of describing the effects of islands or stochastic regions on plasma confinement. The source-free treatment of the kinetic equation is also a limitation, particularly in the case of strong local heating of trapped electrons by $\mathrm{X} 2$ electron cyclotron waves. Even for $\mathrm{O} 1$ heating of passing electrons, one must expect the high-energy portion of the distribution function to deviate substantially from $f_{\mathrm{m}}$. For the ECH discharges described in this paper, one must therefore consider the neoclassical values of $\Gamma_{\mathrm{e}}$ to represent a lower limit on the electron particle fluxes.

\section{The Four Helical Devices}

\section{A. Configurations}

Both CHS (with 8 field periods) and LHD (with 10 field periods) have magnetic configurations of the $l=2$ heliotron type [31]. The rotational transform, $l / 2 \pi$, is fairly flat in the central part and increases to the edge. The low-order rational $\imath / 2 \pi=1 / 2$ is in the confinement region and can play an important role for CERC. In LHD, the $m / n=2 / 1$ island size can be controlled by external coils. The different magnetic configurations discussed here are obtained by inward- or outward shifts, characterised by the major radius $R$ : for CHS $0.921<R<0.974 \mathrm{~m}$ and $3.50<R<3.90 \mathrm{~m}$ for LHD. In a poloidal cross-section, $B$ has a saddle-point, a feature typical of heliotrons. The saddle-point is close 
to the magnetic axis in the $R=0.974 \mathrm{~m}$ and in the $R=3.90 \mathrm{~m}$ configuration for CHS and LHD, respectively. In both helical devices, the toroidal ripple on axis is fairly small for the inward-shifted configurations, but increases with outward-shift. All configurations have only fairly small elongation (roughly, the toroidally averaged flux surfaces correspond to a circular cross-section). Inward-shift leads to a reduction of the effective ripple, $\varepsilon_{\text {eff }}$, whereas an outward-shift results in a strong increase of $\varepsilon_{\text {eff }}$. In a simple picture, the variation of the minima of $B$ on the flux surface is decreased (increased) for an inward-shift (outward-shift), and only the component of $\nabla B$ in the helical direction determines the radial $\nabla B$-drift (in the bounce-averaged picture). Consequently, inward-shifted heliotron configurations are drift-optimised (in the sense of the so-called $\sigma$ optimisation [32]) and outward-shifted de-optimised. This drift-optimisation of the deeply trapped particles for LHD was also analysed in Ref. [25, 33].

TJ-II is a flexible heliac with 4 field periods where the bean-shaped flux surfaces rotate around the central conductor, composed of a circular and a helical coil. The ratio of the currents in the central conductors allows TJ-II to change the plasma position, shape and size to some extent [34]. The rotational transform profile is fairly flat (low shear) and can be changed from $\imath / 2 \pi=0.9$ to 2.5 . In general, $B$ increases towards the central conductor [35]. The CERC discharges described here are performed only in the "standard" configuration with $\imath(0) / 2 \pi \approx 3 / 2$, a small inductive current is applied to vary $\imath(0) / 2 \pi$ and to analyse the impact of the $m / n=2 / 3$ island on the confinement. For the ECH plasmas well into the $\operatorname{lm} f p$ regime, neoclassical losses are dominated by rapidly drifting particles trapped in localised ripples in the magnetic configurations [24]. On axis, $B$ varies by about $3 \%$ (leading to $\varepsilon_{\text {eff }}(0) \approx 0.03$ ), and $\varepsilon_{\text {eff }}$ strongly increases with radius. In this inter-machine analysis, TJ-II represents the maximum $\varepsilon_{\text {eff }}$ configuration [27]. 
The magnetic configuration of W7-AS is partly drift-optimised due to an (average) elongation of about 2. W7-AS was designed following the "linked mirror" concept where straight parts are linked together in a rather elongated region (where the flux surfaces have approximately an elliptical cross-section) with strong local toroidal curvature. The magnetic axis of W7-AS with 5 field periods is similar to a (smoothed) pentagon. This (averaged) elongation reduces both the Shafranov shift and the neoclassical transport in the Pfirsch-Schlüter as well as in the plateau regime, but not in the $\operatorname{lmfp}$ regime where localised ripples dominate. With increasing $\imath / 2 \pi$ (and outward-shift), the localised minima of $B$ deepen, in particular at outer radii, and dominate the $\operatorname{lm} f p$ neoclassical transport. In the CERC discharges, mainly low- $\iota / 2 \pi$ configurations were used. In addition to the inward-shift, a separated coil current supply allows for an independent variation of the toroidal mirror term controlling the power absorption for trapped electrons for ECH X2-mode. The W7-AS "standard" configuration at $t(0) / 2 \pi \approx 1 / 3$ has a fairly pronounced minimum of $B$ in the ECH launching plane close to the elliptical cross-section. The effective ripple is moderate in the central part $\left(\varepsilon_{\text {eff }}(0) \leq 0.015\right)$ and increases only slowly with radius compared to the other configurations. Finally, the vacuum rotational transform is flat (low shear), but W7-AS was typically operated with inductive feed-back control of the plasma current resulting in a strongly reduced $l(0) / 2 \pi$ (with significant shear) in the discharges included in the CERC collaboration within the ISPDB activity.

\section{B. ECH operation modes and deposition}

In the heliotron magnetic field topology of CHS and LHD (Sec. 3A), a peaked ECH deposition close to the magnetic axis is difficult to achieve. The heliotron windings prohibit the optimum ECH launch (located in the vertically elongated cross-section) perpendicular to $B$ for the inward-shifted configurations; a very similar situation existed in the ATF torsatron and is well documented in Ref. 
[36]. Moreover, the angle between the rays and the $B=$ const. contours is fairly small $[37,38]$ (small gradient of $B$ along the rays) leading to high absorption, and the deposition is very sensitive to small changes of $B$, but also to the local electron temperatures due to the relativistic down-shift of the resonant cyclotron frequency (this effect is expected to be very important for the CERC scenarios at LHD with the very high $T_{\mathrm{e}}$, but low $n_{\mathrm{e}}$ ). An "anomalous dispersion" effect [39], which can lead to the bending of the rays away from the resonance and is only obtained in the tracing with the weakly relativistic (hot) dispersion [40], is negligible for the low densities in LHD. Ray-tracing calculations [38, 41] for the LHD inward-shifted configurations (with fairly high $B$-gradient along the rays, i.e. optimum localisation conditions) gives almost complete absorption (for proper polarisation) within the central $20 \%$ of the plasma radius. The deposition is close to the maximum of $B$ on the flux surfaces and absorption by ripple-trapped electrons can be neglected. For the outward-shifted configurations (with the magnetic axis close to the saddle-point), the situation is different: highly peaked on-axis deposition cannot be obtained for realistic conditions, furthermore, absorption by ripple-trapped electrons cannot be ignored.

In the CHS discharges, only 1 gyrotron with $200 \mathrm{~kW}$ power, $53.2 \mathrm{GHz}$ in X2-mode corresponding to a resonant $B=0.95 \mathrm{~T}$ was available. In addition, 2 (positive) neutral beams with $800 \mathrm{~kW}$ injection power each were used. For the very low densities (less than $5 \times 10^{18} \mathrm{~m}^{-3}$ ), however, only a small fraction of the NBI power is absorbed [42].

$\mathrm{ECH}$ at 82.7 and $84.0 \mathrm{GHz}[38]$ with up to $1.1 \mathrm{MW}$ power were available at LHD in the CERC experiments. The resonant field is $B \approx 3 \mathrm{~T}$ and $\approx 1.5 \mathrm{~T}$ for $\mathrm{O} 1$-mode and X2-mode, respectively (a direct comparison of O1- and X2-mode with the new $168 \mathrm{GHz}$ gyrotrons in discharges at the same $B$ is missing here). At LHD, negative-ion-based NBI (energies of 160 to $180 \mathrm{keV}$ ) with tangential injection and injection power up to $10 \mathrm{MW}$ was applied in addition to the ECH. For the rather low 
densities in the CERC experiments, a rather small fraction of the injected power is absorbed almost exclusively by electrons [41].

Both for TJ-II and W7-AS, the ECH is launched from the low-field-side in a more "traditional" way, i.e. roughly with the maximum $B$-gradient along the rays allowing for high localisation of the deposition. For TJ-II, an additional (steerable) mirror was placed in the vacuum vessel to allow for this launching because of its strong helical plasma shape. For both devices, the launching is nearly independent of the different magnetic configurations (only the resonant $B$ must be slightly adjusted), and highly peaked deposition profiles are obtained from ray-tracing calculations [35, 43]. At TJ-II, part of the power for X2-mode is absorbed by ripple-trapped electrons ( $B$ on axis varies by about $3 \%$, and the deposition is roughly at the $B$-minimum). For the large ripples in TJ-II [27], trapped electrons at higher energy can escape in the low- $n_{\mathrm{e}}$ discharges without a substantial radial electric field [44]; see Sec.2. High-frequency ECH power modulation experiments indicate a broadening of the power deposition profile compared to ray-tracing calculations. W7-AS is more flexible, magnetic configurations with a significant $B$-minimum in the launching plane (e.g. $B$ on axis varies by more than $4 \%$ in the low- $\iota / 2 \pi$ "standard" configuration) and with a $B$-maximum allow for the analysis of the ripple-trapped electron heating in the X2-mode discharges (for O1mode, direct heating of ripple-trapped electrons is negligible in all configurations). Also at W7-AS, a broadening of the ECH power deposition profile was found by high-frequency power modulation for both O1- and X2-mode [45]. Direct losses in scenarios with significant absorption of X2-mode by ripple-trapped electrons can be excluded as demonstrated by global Monte Carlo simulations [46].

TJ-II is equipped with 2 gyrotrons at $53.2 \mathrm{GHz}$ with about $200 \mathrm{~kW}$ power each operating in X2mode at a resonant $B \approx 0.95 \mathrm{~T}$. In the early phase, W7-AS was operated with ECH at $70 \mathrm{GHZ}$ in 
O1-mode for resonant $B \approx 2.5 \mathrm{~T}$ with up to $800 \mathrm{~kW}$ power. Later on, $\mathrm{ECH}$ at $140 \mathrm{GHz}$ in X2-mode ( $B \approx 2.5 \mathrm{~T}$ ) with up to $1.6 \mathrm{MW}$ was available. For both TJ-II and W7-AS, purely EC heated discharges were included in the CERC analysis.

\section{Diagnostic equipment relevant to CERC}

Thomson scattering diagnostics are available at these four helical devices, at LHD and TJ-II with very high radial resolution. Except for LHD (where the $n_{\mathrm{e}}$-profiles are obtained from interferometry), $n_{\mathrm{e}^{-}}$and $T_{\mathrm{e}}$-profiles are based on this diagnostic. The ECE diagnostic at LHD (recently with absolute calibration), TJ-II and W7-AS supports the $T_{\mathrm{e}}$-profiles, but yields in addition also information on fast transitions. In heliotron configurations with a small $B$-gradient close to the axis, the radial resolution of the ECE diagnostic becomes rather poor, this effect is equivalent to the poor localisation of the ECH deposition; see Sec. 3B. Fast HIBP data are available only at CHS and TJ-II for the profile of the electrostatic potential, $\Phi(r)\left(E_{\mathrm{r}}=-\Phi^{\prime}\right)$. For very low densities, the HIBP intensity is proportional to the local density, allowing conclusions on fast transitions in both $\Phi$ and $n_{\mathrm{e}}$. Interferometry is not suited for the resolution of small changes in the density profile within the CERC region. Only at TJ-II, does the $T_{\mathrm{e}}$ from ECE lead to a complete documentation of fast transitions. Active CXRS is used at LHD as well as at W7-AS to determine the poloidal rotation velocity and the radial electric field (with a rather poor quality at W7-AS partly due to the low intensity of the diagnostic NBI). The analysis of CERC discharges is supported also by other standard diagnostics (e.g. the energy content from diamagnetic loops) which are less important in this context.

\section{The CERC Findings}




\section{A. Profile documentation}

For the four helical devices, Figure 1 shows the typical feature of the $T_{\mathrm{e}}$-profiles for CERC at quite different $n_{\mathrm{e}}, P_{\mathrm{ECH}}(\mathrm{ECH}$ power), and $B$. Here, the normalized minor radius, $\rho$, is defined by $\rho=r / a$, with $a$ being the averaged plasma minor radius. Within the CERC region, $T_{\mathrm{e}}$ is highly peaked in all devices. This feature is found in the range $n_{\mathrm{e}} \approx 0.15 \times 10^{19} \mathrm{~m}^{-3}$ (at LHD) to $n_{\mathrm{e}} \approx 5.3 \times 10^{19} \mathrm{~m}^{-3}$ (at W7-AS). With increasing density at fixed $P_{\mathrm{ECH}}$, however, the peaking of $T_{\mathrm{e}}$ profile becomes less pronounced (compare Fig. 4). Figure 2 documents the establishment of CERC due to $P_{\mathrm{ECH}}=0.78$ MW adding to the NBI sustained target plasma with an absorbed power $P_{\mathrm{NBI}} \approx 1.3 \mathrm{MW}$ at LHD [47] $\left(n_{\mathrm{e}} \approx 0.3 \times 10^{19} \mathrm{~m}^{-3}, B \approx 1.5 \mathrm{~T}, R_{\mathrm{ax}}=3.8 \mathrm{~m}\right)$. For these conditions, the power threshold for CERC establishment was found to exceed $P_{\mathrm{ECH}}>0.58 \mathrm{MW}$. The peaked central $T_{\mathrm{e}}$-profile (Fig. 2(a)) with the additional ECH corresponds to the peak in the $E_{\mathrm{r}}$-profile (Fig. 2(b)) roughly in the same radial range which is missing for the pure NBI case. The $T_{\mathrm{i}}$-profiles are roughly identical for both cases without showing any peaking. Outside of the CERC region, $T_{\mathrm{i}} \approx T_{\mathrm{e}}$ is found. The very large $\varepsilon_{\text {eff }}$ leads also to an electron-root at the outer radii for these very low collisionalities (see also [28]).

The existence of a threshold for the CERC formation with respect to ECH power and density has been demonstrated in all four helical devices. This finding indicates that the CERC formation is based on a bifurcation mechanism. In particular, thresholds in the ECH power have been identified in CHS [42], in LHD [19, 47, 48] and in W7-AS [49]. Figure 3 shows such an ECH power scan for LHD [19] ( $P_{\mathrm{ECH}} / n_{\mathrm{e}}$ is used here since the central $n_{\mathrm{e}}$ was not feed-back controlled). In Fig. 3(a), the normalized scale length of the $T_{\mathrm{e}}$-gradient versus $P_{\mathrm{ECH}} / n_{\mathrm{e}}$ shows a clear jump at the threshold. The other plots, the corresponding $T_{\mathrm{e}}$ and the normalised electron heat diffusivity, $\chi_{\mathrm{e}}(r)$, from the experimental power balance, also demonstrate this bifurcation feature. The $P_{\mathrm{ECH}}$-threshold is found 
to increase with $n_{\mathrm{e}}[17,41,48]$. Consequently, the $n_{\mathrm{e}}$-threshold for fixed $P_{\mathrm{ECH}}$ is also documented for LHD [48] and for TJ-II [18, 50].

A density scan with $P_{\mathrm{ECH}} \approx 1.2 \mathrm{MW}$ in X2-mode at W7-AS for the "standard" configuration is shown in Figure 4. Note, that the densities here are much higher than the typical ones in the CERC discharges in the other helical devices. The CERC feature is marginal at the highest density $\left(7 \times 10^{19} \mathrm{~m}^{-3}\right)$. The electron power balance analysis (see Sec. 4D) shows very good agreement with the neoclassical prediction for the ion-root (outside of the CERC region) up to $2 / 3$ of the minor radius. Where $E_{\mathrm{r}}$ is small in magnitude, the neoclassical electron heat diffusivity is determined by the $1 / \mathrm{v}$-regime, and the electron heat flux becomes independent of density leading roughly to the same $T_{\mathrm{e}}$-profiles in the density scan. In the CERC region, the peaking of the $T_{\mathrm{e}}$-profiles is reduced for increasing density. In this scan, the energy confinement time scales linearly with $n_{\mathrm{e}}$ which is a clear deviation from the stellarator ISS04 scaling law, $\tau_{E} \propto n_{\mathrm{e}}^{0.54}[51]$.

\section{B. Impact of magnetic configuration and ECH mode}

The effect of the magnetic configuration, in particular of the effective ripple as well as of the ECH power absorption by ripple-trapped electrons in the CERC region has been analysed in LHD and in W7-AS (see also Sec. 3). At W7-AS, ECH power and density scans had been performed at $B=2.5 \mathrm{~T}$ both for O1-mode [52] and X2-mode [49]. The ECH in O1-mode is nearly completely absorbed by passing electrons, and the effect of $\varepsilon_{\text {eff }}$ for different magnetic configurations on the CERC formation could be analysed. It turns out, that for equivalent $P_{\mathrm{ECH}}$ and $n_{\mathrm{e}}, \mathrm{CERC}$ is more easily established for larger $\varepsilon_{\text {eff }}$ as predicted by neoclassical theory (see Sec. 2), i.e. the threshold with respect to $P_{\mathrm{ECH}}\left(n_{\mathrm{e}}\right)$ is lower (higher). The direct comparison of the O1- and the X2-mode 
discharges at the same $P_{\mathrm{ECH}}$ and $n_{\mathrm{e}}$ is not available for the profile documentation, however the significant power absorption by ripple-trapped particles and the resultant "convective" contribution to the electron flux was confirmed by the faster $T_{\mathrm{e}}$-decay after the ECH was switched off [52]. For the X2-scenario, both ECH power and density scans were performed [49] for two magnetic configurations: with a minimum of $B$ of about $4.5 \%$ ("standard" configuration) and with a maximum of $B$ in the ECH launching plane. Both the density scan (at fixed $P_{\mathrm{ECH}} \approx 1.2 \mathrm{MW}$ ) and the power scan (at fixed $n_{\mathrm{e}} \approx 2 \times 10^{19} \mathrm{~m}^{-3}$ ) confirmed that with the additional "convective" flux in the "standard" configuration, CERC is easier to establish. Furthermore, Monte Carlo simulations (by the GNET code [53]) showed that this ECH-driven flux is of comparable magnitude with the ambipolar flux in the ion-root region [49] thus affecting the transition to the electron-root in the core.

In LHD, the CERC formation was analysed in a $R_{\mathrm{ax}}$-scan with $3.60,3.75$ and $3.90 \mathrm{~m}$ [41] reflecting the configurational flexibility. As described in Sec. 3B, the ECH deposition is affected within such a $R_{\mathrm{ax}}$-scan. For both inward-shifted configurations with $n_{\mathrm{e}} \approx 0.4 \times 10^{19} \mathrm{~m}^{-3}$, a clear CERC feature is found whereas for $R_{\mathrm{ax}}=3.90 \mathrm{~m}$ with slightly higher density $\left(n_{\mathrm{e}} \approx 0.6 \times 10^{19} \mathrm{~m}^{-3}\right)$ CERC appears to be only marginal. In this scan, however, both $P_{\mathrm{ECH}}$ and (absorbed) $P_{\mathrm{NBI}}$ are also different $\left(0.53<P_{\mathrm{ECH}}<0.94 \mathrm{MW}\right.$ and $\left.0.9<P_{\mathrm{NBI}}<2.4 \mathrm{MW}\right)$. The $R_{\mathrm{ax}}=3.90 \mathrm{~m}$ configuration has a significantly higher $\varepsilon_{\text {eff }}$ compared to the inward-shifted configurations (see Sec. 3A), and from the neoclassical prediction (Sec. 2), it should be easier to establish CERC. The experimental results, however, appear contrary. With the saddle-point in $B$ close to the magnetic axis in this configuration (see Sec. 3B), an uncertainty in the location of the ECH absorption exists, in particular if the $84.0 \mathrm{GHz}$ should be deposited very close to the axis. Further analysis (with e.g. the 
hot dispersion taken into account) of the ECH deposition in this $R_{\mathrm{ax}}$-scan is needed to increase the accuracy and, consequently, to allow for a final conclusion.

\section{The radial electric field}

Large positive $E_{\mathrm{r}}$ is observed in the CERC region in the four helical devices. These findings are consistent with neoclassical predictions based on the ambipolarity of the radial particle fluxes; see Sec. 2. Figure 5 shows examples of the measured $E_{\mathrm{r}}$ in comparison to the neoclassical ambipolar $E_{\mathrm{r}}$ calculated with the measured density and temperature profiles. For CHS, the analytic expressions for neoclassical radial fluxes of Ref. [54] were used. In the LHD example (see Fig. 5(b)), the $E_{\mathrm{r}}$ was derived from DCOM calculations [25]. The Monte Carlo code MOCA [24] was used at TJ-II, and DKES [23] for W7-AS. The mono-energetic transport coefficients calculated by these codes are the basis for the energy convolution to obtain the thermal transport coefficients and, with the gradients from fits to the experimental profiles, the particle and energy fluxes. The discrepancy between both $E_{\mathrm{r}}$ profiles in the TJ-II example is partly attributed to the uncertainty of measured density and temperature profiles (new comparisons are in progress with improved HIBP and other profile diagnostics). In the W7-AS example, several roots are found from the ambipolarity condition in the local approach, and the transition between the electron- and the ion-root (solid line) is obtained from the diffusion equation for $E_{\mathrm{r}}$ [30] which corresponds to the Euler-Lagrange form of the generalised heat production rate; see Sec. 2. Here, the diffusion coefficient for $E_{\mathrm{r}}$ (corresponding to a poloidal shear viscosity) was simply assumed so that the width of the transition layer is roughly consistent with the one obtained at CHS from the HIBP measurements; comp. Sec. 4E. As an important conclusion of Fig. 5, the radial bifurcation nature of the ambipolar $E_{\mathrm{r}}$ is confirmed to be the common physical mechanism in the CERC findings in the four helical devices. 
Finally, the impact of a "convective" contribution to the electron flux for X2-scenarios with significant ECH absorption by ripple-trapped electrons is briefly discussed. GNET Monte Carlo simulations [53] for W7-AS discharges indicate a weaker dependence of these "convective" contributions on $E_{\mathrm{r}}$ compared to the "diffusive" neoclassical electron flux. For the W7-AS and LHD scenarios analysed, the "convective" contributions were roughly comparable to the "diffusive" ionroot particle fluxes and should be taken into account in the ambipolarity condition. Such a selfconsistent modeling, however, is lacking so far. Straight-forward Monte Carlo techniques are extremely time consuming and not well suited for such an approach; the mapping technique [55] offers a realistic chance for a self-consistent treatment of both "convective" and "diffusive" flux simulations. Furthermore, the violation of the assumptions used for the derivation of the local mono-energetic diffusion coefficients (see Sec. 2) for very large $\varepsilon_{\text {eff }}$ can also lead to "convective" flux contributions as was shown in [44] for TJ-II. All these additional effects should result in an increase of the electron fluxes in the ambipolarity condition and, consequently, to larger $E_{\mathrm{r}}$ in the CERC region. However, a detailed analysis of the particle balance with the sources from recycling and gas puffing is currently lacking for the CERC scenarios.

\section{Electron energy balance analysis}

The electron energy balance has been intensively analysed for CERC discharges and compared with the neoclassical prediction in the four helical devices (e.g. for CHS in [42], for LHD in [47, 56], for TJ-II in [18], and for W7-AS in [49, 52]). It should be mentioned here, that the neoclassical analyses in CHS and LHD, which were based on an analytical model [5], will be repeated with DCOM [25] and/or GSRAKE [57] in the near future, and are not shown here. And a second remark: the current paper follows the "traditional" picture where a purely "diffusive" ansatz for the electron 
heat flux density, $Q_{\mathrm{e}}=-n_{\mathrm{e}} \chi_{\mathrm{e}} T_{\mathrm{e}}^{\prime}$, is used for the experimental power balance, and $\chi_{\mathrm{e}}(r)$ (the "experimental" electron heat diffusivity) is compared with the neoclassical heat diffusivity (the diagonal term, $D_{3}^{\mathrm{e}}-3 D_{2}^{\mathrm{e}} / 2$, in the neoclassical transport matrix related to $T_{\mathrm{e}}^{\prime}$ as the "thermodynamic force" [23]). This ansatz is reasonable for the neoclassical $1 / v$-regime with only small $E_{\mathrm{r}}$, but within the CERC region the large positive $E_{\mathrm{r}}$ (in combination with the off-diagonal term) counteracts the $T_{\mathrm{e}}{ }^{\prime}$-driven heat flux. Consequently, only the flux densities from the experimental balances (by integrating the sources and sinks for stationary conditions) should be compared with the neoclassical predictions.

Figure 6 shows the comparison of the experimental $\chi_{\mathrm{e}}(r)$ (error bars in Fig. 6(a) are obtained by least-squares fitting of the power balance [30]) with the neoclassical heat diffusivity with the ionand electron-root $E_{\mathrm{r}}$ included and, as a reference, for $E_{\mathrm{r}}=0$ (for the comparison of the ambipolar $E_{\mathrm{r}}$ with the experimental one and the position of the transition layer, see Fig. 5). As one of the main criteria for CERC, $\chi_{\mathrm{e}}$ must be much smaller than the neoclassical one for $E_{\mathrm{r}}=0$ within the CERC region which is clearly the case in this example. Furthermore, $\chi_{\mathrm{e}}$ is in good agreement up to about $2 / 3$ of the minor radius with the neoclassical prediction for the ion-root. The electron-root heat diffusivity, however, is significantly lower than the experimental $\chi_{\mathrm{e}}$ within the CERC region. In this series of discharges with $P_{\mathrm{ECH}}=770 \mathrm{~kW}$ in X2-mode and at $n_{\mathrm{e}} \approx 2 \times 10^{19} \mathrm{~m}^{-3}$, part of the power is absorbed by ripple-trapped electrons in the launching plane (comp. Sec. 3A) leading to significant "convective" flux contributions [49, 53]. Although these contributions for the electron heat flux have not been calculated, a rough estimate (with an averaged heat flux contribution at twice the thermal velocity) leads to a value of about $200 \mathrm{~kW}$. Taking all the uncertainties into consideration, in particular a broadening of the $\mathrm{ECH}$ deposition profile (as indicated by high- 
frequency power modulation experiments) and the violation of the basic assumptions of the neoclassical approach, any conclusion on "anomalous" transport contributions within the CERC region is not justified.

At W7-AS, equivalent experiments in O1-mode where the ECH power is absorbed by passing electrons show also a clear underestimation of the (purely) neoclassical heat diffusivity compared to the experimental $\chi_{\mathrm{e}}$ within the CERC region [52] at low density, however this discrepancy is reduced at higher densities. These indications are consistent within a kinetic (and neoclassical) picture where the deviation from the Maxwellian is reduced with increasing density. Here, it should be mentioned again, that the densities in the CERC discharges at W7-AS are much higher than in the other helical devices.

The theoretical (and numerical) tools must be significantly improved to allow for a quantitative conclusion on additional "anomalous" transport contributions related to turbulence. For these CERC conditions with peaked ECH deposition and low densities, any local approach is insufficient. In particular, quasi-linear degradation effects (or even non-linear ones) for the description of the ECH deposition must be included in the kinetic modeling in full 5D-phase space, but such a treatment is not-at-all trivial.

\section{E. A Question of Cause and Effect}

Here, the shear layer in the poloidal rotation, i.e. the narrow transition region between ion- and electron-root, and its interpretation as an internal transport barrier (ITB) is briefly discussed. Figure 7 shows the profiles of (a) the electrostatic potential measured by HIBP with high radial resolution and (b) the derived $E_{\mathrm{r}}$ at CHS [14] for a CERC scenario. These figures are the CHS counterpart of those shown in Fig. 2. Even in the early phase of the CERC findings, the $E_{\mathrm{r}}$ profiles have been 
discussed only in terms of multiple roots of the neoclassical ambipolarity for stellarators in the lmfp-regime; additional "anomalous" (i.e. turbulent) contributions to the particle fluxes have been assumed to be intrinsically ambipolar.

Within the shear layer, the density fluctuations also derived from the HIBP data (see Fig. 7(c)) are moderately decreased. This finding has been interpreted $[14,58]$ as the formation of an ITB triggered by the neoclassical transition in the ambipolar $E_{\mathrm{r}}$, and the reduction of "anomalous" transport driven by turbulence within the ITB has been attributed to the strong shear in the poloidal flow. (It should be noted, that the shear in the poloidal flow responsible for turbulence suppression is $r\left(E_{\mathrm{r}} / r\right)^{\prime}$ and not simply $E_{\mathrm{r}}^{\prime}$.) This picture leads to higher $T_{\mathrm{e}}$ gradients within the narrow ITB, but not to the highly peaked $T_{\mathrm{e}}$-profile within the CERC region (for a fairly similar discussion of LHD and JT-60U findings, see Ref. [3]).

After more detailed analysis of the electron energy balance in comparison with neoclassical predictions (see Sec. 4D), a "tokamak-based" interpretation with ITB formation being responsible for a significant reduction of the turbulent transport within the CERC region cannot be supported in the four helical devices. Even allowing for all inaccuracies of the "traditional" neoclassical modeling, the strongly positive electron-root $E_{\mathrm{r}}$ is the cause of the significantly improved confinement and the highly peaked $T_{\mathrm{e}}$-profiles. A reduced fluctuation level as shown in Fig. 7(c) is an effect of the strongly sheared poloidal flow within the narrow transition layer and may have the additional benefit of reducing turbulent transport contributions.

\section{F. Transitions and bifurcations}

Spontaneous transitions (without external disturbances) in CERC have been observed at CHS [13, 14, 59-61] (also called bifurcations and electric pulsations) in the electric potential measurements. At W7-AS, nearly the identical feature was found in the electron temperature $[49,58]$. In both 
cases, the time scale of these transitions was very short (less than $1 \mathrm{~ms}$ ), and the decay time was roughly $1 / 3$ of the one for establishing CERC. However, proof was lacking that the transitions in the potential and in the $T_{\mathrm{e}}$-profile are identical. These very fast phenomena have only been observed for conditions close to the CERC threshold. At W7-AS, a $B$-scan has been performed shifting the ECH deposition slightly off-axis (both to the low- and high-field side); here, the Shafranov shift [62] for the highly peaked pressure profiles and the relativistic frequency shift in the $\mathrm{ECH}$ absorption [49] have been found to be important for establishing CERC.

The ultimate proof, that the fast transitions both in the potential and in the electron temperature profile reflect the physics of CERC, has been obtained at TJ-II [10] from the combined analysis of HIBP and ECE data; see Figure 8. The time traces are nearly identical, only a slightly slower $T_{\mathrm{e}}$ variation is indicated which is in agreement with neoclassical simulations of the ECH switch-off at W7-AS [49]. In addition, the impact of the CERC transitions on the particle balance is documented in Fig. 8: the density (linearly related to the HIBP intensity) is affected in the transitions, anticorrelated to both the potential and $T_{\mathrm{e}}$. This is a very important finding (which cannot be derived from other diagnostics) indicating that the $n_{\mathrm{e}}$-profile becomes hollower with CERC. This implies that the $T_{\mathrm{e}}{ }^{\prime}$-driven contribution (outward pinch) exceeds the $E_{\mathrm{r}}$-driven one (inward pinch) in the electron particle balance resulting in an inward diffusion driven by $n_{\mathrm{e}}$ ' for compensation. This simple picture might be in conflict with the ion particle balance $\left(n_{\mathrm{i}}^{\prime} / n_{\mathrm{i}}\right.$ is much smaller than $E_{\mathrm{r}} / T_{\mathrm{i}}$ in the CERC region), however a more detailed particle balance analysis with the particle sources included is needed to determine whether this is the case. Finally, no effects of the transitions on the

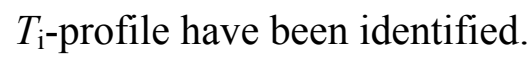

The transient behavior of $E_{\mathrm{r}}(r . t)$ at CHS allows for conclusions on the bifurcation physics [61]. The time derivative of $E_{\mathrm{r}}$ is related to a radial current (i.e. a short violation of the ambipolarity 
condition) during the transitions which can be compared with the neoclassical particle fluxes. It turns out, that this radial current is roughly consistent with the unbalanced neoclassical particle fluxes. At W7-AS (without fast $E_{\mathrm{r}}$ data), the phase of the ECH switch-off has been simulated with the time-dependent diffusion equation for $E_{\mathrm{r}}$ for ECH in O1-mode [52] as well as in X2-mode [49]. The conclusions from CHS could be confirmed: the neoclassical particle fluxes (including additional "convective" contributions for X2-mode) are consistent with the fast $T_{\mathrm{e}}$-decay found in the ECE data. In summary, the nonlinearity in the neoclassical ambipolarity condition is the origin of the bifurcation nature of CERC close to thresholds. Finally, ECH power modulation experiments with stimulated transitions at W7-AS [49] also confirmed the $T_{\mathrm{e}}$-dependence of this bifurcation picture.

\section{G. Impact of islands on CERC formation}

Finally, the impact of islands located at low-order rational values in the rotational transform profiles on the CERC formation is briefly discussed. Both at LHD [41] and TJ-II [20, 63], indications are found that the thresholds to establish CERC are reduced by pronounced islands $(m / n=2 / 1$ at $\iota / 2 \pi=1 / 2$ at LHD and $m / n=2 / 3$ at $\iota / 2 \pi=3 / 2$ at TJ-II). A detailed analysis resolving the island width and location is still lacking, the (transient) current densities (i.e. ohmic, bootstrap and neutral beam current drive, NBCD) must be calculated and the current diffusion must be taken into account. With the reasonable assumption that pronounced islands lead to a locally increased electron transport (parallel transport in the x-point region), their existence within the CERC region can be excluded. On the other hand, these islands might limit the radial extension of CERC and might be located closely outside the shear layer ("foot-point" [41]). 
Two basic mechanisms are described in the following which have been discussed for electron ITBs in tokamaks related to the presence of rational magnetic surfaces (see e.g. Refs. [64, 65] for comparison with the experimental findings at RTP). The first mechanism [66] (for a more fundamental approach, see Ref. [67]) is related to parallel transport in the near vicinity of an island. Here, only high-order rationals in $\imath(r) / 2 \pi$ exist and both island overlapping and ergodisation can be suppressed for a broad spatial spectrum of the perturbed field (i.e. high poloidal Fourier mode numbers) depending on the shear, $(l(r) / 2 \pi)^{\prime}$. In these narrow regions close to the low-order rational magnetic surface, improved confinement can be obtained, i.e. an electron ITB. In Ref. [66] with the 3D-MHD modeling, the impact of a radial electric field was disregarded, nevertheless, this model can also be applied for helical devices. Parallel electron transport in the x-point region affects the ambipolarity condition and might increase $E_{\mathrm{r}}$ thus lowering the CERC threshold. Within the neoclassical picture, however, the radial transport is dominated by the ripple-trapped electrons which are not affected by the islands.

The second mechanism [68] "introduces" stellarator neoclassical transport in the lmfp regime into the tokamak theory. The flux surfaces close to the island separatrix become corrugated leading to a "local" ripple. This effect might be important for tokamaks where the ambipolarity condition becomes nonlinear (with the same bifurcation nature as described in Sec. 4F). For helical devices, this effect of corrugated flux surfaces is not so clear. For a strong radial variation of $\varepsilon_{\text {eff }}$, a quantitative analysis must be performed. For example, the mono-energetic diffusion coefficient was calculated with the NEO code for Uragan-3M in case of magnetic islands [69]: a significant increase of $\varepsilon_{\text {eff }}$ close to the island was found. Consequently, an increased $\varepsilon_{\text {eff }}$ supports establishment of CERC, i.e. reduces the threshold. 


\section{Summary and Conclusions}

Core "electron-root" confinement is found in CHS, LHD, TJ-II and W7-AS with very similar signatures. CERC is clearly identified as an improved electron energy confinement related to the transition to the "electron-root" solution of the ambipolarity of the neoclassical particle fluxes. The neoclassical bifurcation picture is confirmed in spontaneous transitions where the central electrostatic potential (obtained from HIBP) changes in an equivalent manner to the central electron temperature (measured by ECE) and anti-correlated to the central density (proportional to the HIBP intensity at low $n_{\mathrm{e}}$ ). These important findings in TJ-II are only partly supported by the other devices where data from one of these diagnostics is lacking.

Configurations with a large effective helical ripple, $\varepsilon_{\text {eff }}$, in the central region allow for an easier access to CERC. This neoclassical prediction is confirmed both by configuration scans in W7-AS and by the inter-machine comparison. In particular, TJ-II with the fairly large $\varepsilon_{\text {eff }}(0) \approx 0.03$ (strongly increasing with radius) finds CERC at fairly low $T_{\mathrm{e}}$ (and also at low ECH power) whereas CHS (in the "standard" configuration with $R=0.92 \mathrm{~m}$ ) with $\varepsilon_{\text {eff }}(0) \approx 0.004$ clearly achieves CERC at higher $T_{\mathrm{e}}$ together with a broader profile (at equivalent ECH power). An accurate documentation of the density and power thresholds for the transition to CERC depending on $\varepsilon_{\text {eff }}$ is missing, so far. However, such configuration scans are not unambiguous in all devices. For example, the (inwardshifted) LHD configurations with $R_{\text {ax }} \approx 3.53,3.60$ and $3.75 \mathrm{~m}$ have fairly low $\varepsilon_{\text {eff }}(0) \leq 0.005$ in the ECH deposition zone close to the axis, whereas $\varepsilon_{\text {eff }}(0) \geq 0.015$ for the $R_{\text {ax }} \approx 3.90$ m configuration but the region with $|\nabla B| \approx 0$ (saddle-point in $B$ ) prevents a highly peaked ECH deposition close to the axis. 
For ECH in X2-mode with significant absorption by ripple-trapped electrons, additional "convective" fluxes can reduce the density and power thresholds for CERC, as is found in the W7AS configuration scans. For O1-mode, the ECH power is nearly completely absorbed by passing electrons but, nevertheless, an enhanced transport is expected due to the deviation at higher energies of the electron distribution function from the Maxwellian, in particular at low densities. A density scan for O1-mode at W7-AS indicates that the heat diffusivity from the experimental power balance becomes closer to the neoclassical prediction within the CERC region as the density increases.

Within CERC, no accumulation of impurities is expected, the strongly positive $E_{\mathrm{r}}$ pushes the impurities outward. This prediction is supported by the high density CERC in W7-AS. However, a narrow CERC is not sufficient at higher densities, ELMs (edge localized modes) at outer radii are also necessary as all quiescent H-mode discharges show strong impurity accumulation (for intermediate or low densities outside the separatrix; impurity screening in the SOL is obtained for very high densities). This advantage of positive $E_{\mathrm{r}}$ leads to the question of how to extend the radial CERC range, in particular at high densities for which typically strongly negative $E_{\mathrm{r}}$ are found [16] at outer radii (within the density gradient region). At high densities, the strong collisional power transfer, $P_{\mathrm{ei}} \propto n_{\mathrm{e}}^{2}$, leads to $T_{\mathrm{i}} \approx T_{\mathrm{e}}$ with negative $E_{\mathrm{r}}$ outside of CERC. Only within CERC can a highly peaked $E_{\mathrm{r}}(r)$ be established, sustained by sufficient ECH power and decoupled from $T_{\mathrm{i}}$, leading to a rather pessimistic outlook for a broadening of the CERC region.

With respect to stellarator optimisation, quite different conclusions are justified. For quasisymmetric configurations, in particular for the quasi-axisymmetric NCSX, the ECH power threshold is increased by reducing the fraction of ripple-trapped particles (for NCSX, $\varepsilon_{\text {eff }}(0) \approx 0.002$ in the central region). On the contrary, quasi-isodynamic configurations allow for a 
moderate $\varepsilon_{\text {eff }}$ in the center, but decreasing with radius. For example, the configurational flexibility of W7-X allows for a variation of the toroidal mirror term between 0 and about $10 \%$ with $\varepsilon_{\text {eff }}(0) \approx 0.0$ and 0.025 at the axis without affecting a highly peaked ECH deposition. Together with the significant ECH power (up to $10 \mathrm{MW}$ ), CERC presents a fairly optimistic perspective for W7-X even at higher densities.

Finally, this kind of international collaboration with respect to specific physical scenarios in helical devices (well established in the tokamak community) has an important advantage: Not only the helical configurations, but also the heating, the operational regimes, and last-but-not-least the diagnostic equipment are quite different which broadens the "parameter space" for interpretation and modeling. In addition, the reproducibility of specific scenarios (e.g. of confinement modes) in different helical devices essentially improves both the reliability and the possibility of extrapolation towards future configurations (e.g. to a stellarator reactor). Consequently, the international stellarator profile database activity must be extended to additional topics.

\section{Acknowledgments}

The authors would like to acknowledge the teams and groups at CHS, LHD, TJ-II and W7-AS for providing all the experimental data for the current CERC documentation. The continuous discussions with Dr. A. Dinklage, Dr. N. Marushchenko (IPP-Greifswald), Dr. S.Kubo and Dr. Y.Yoshimura (NIFS) are also appreciated. 


\section{References}

[1] R.C. WOLF, “Internal Transport Barriers in Tokamak Plasmas,” Plasma Phys. Contr. Fusion 45, R1 (2003).

[2] J.W. CONNOR et al., "A Review of Internal Transport Barrier Physics for Steady-state Operation of Tokamaks," Nucl. Fusion 44, R1 (2004).

[3] K. IDA et al., "Comparison of Electron Internal Transport Barriers in the Large Helical Device and JT-60U Plasmas,” Plasma Phys. Control. Fusion 46, A45 (2004).

[4] A.A. GALEEV and R.Z. SAGDEEV, "Theory of Neoclassical Diffusion, Chapter2 : Toroidal Stellarators," Review of Plasma Physics 7, 307 (1977).

[5] L.M. KOVRIZHNYKH, "Neoclassical Theory of Transport Processes in Toroidal Magnetic Confinement Systems, with Emphasis on Non-axisymmetric Configurations," Nucl. Fusion 24, 851 (1984).

[6] G. GRIEGER et al., "Physics and Engineering Studies for WENDELSTEIN 7-X," 13th International Conference Plasma Physics Controlled Nuclear Fusion Research, Washington, 1990 (IAEA, Vienna, 1991), Vol. 3, p. 525.

[7] A.F. ALMAGRI, D.T. ANDERSON, and J.N. TALMADGE, “A Helically Symmetric Stellarator (HSX)," IEEE Tran. Plasma Sci. 27, 114 (1999).

[8] G.H. NEILSON, A.H. REIMAN, and M.C. ZARNSTORFF et al., "Physics Issues in the Design of High-beta, Lowaspect-ratio Stellarator Experiments,” Phys. Plasmas 7, 1911 (2000).

[9] S. GORI, W. LOTZ, and J. NÜHRENBERG, "Quasi-isodynamic Stellarators," Theory of Fusion Plasmas (Varenna 1996), Editrice Compositori, Bologna (1996), p. 335.

[10] T. ESTRADA et al., "Electron Internal Transport Barrier Formation and Dynamics in the Plasma Core of the TJ-II Stellarator," Plasma Phys. Control. Fusion 46, 277 (2004).

[11] A. FUJISAWA et al., "Active Trajectory Control for a Heavy Ion Beam Probe on the Compact Helical System," Rev. Sci. Instrum. 67, 3099 (1996).

[12] A. FUJISAWA, S. KUBO, H. IGUCHI, and H. IDEI et al., "Experimental Study of Plasma Confinement and Heating Efficiency through Potential Profile Measurements with a Heavy Ion Beam Probe in the Compact Helical System," 16th International Conference Fusion Energy, Montreal 1996, (IAEA, Vienna, 1997), Fusion Energy 1996, Vol. 2, p. 41$\}$.

[13] A. FUJISAWA et al., "Electric Field Bifurcation and Transition in the Core Plasma of CHS," Plasma Phys. Control. Fusion 40, 627 (1998).

[14] A. FUJISAWA et al., "Transport Barrier Formation and Bifurcation Patterns of Potential Profiles in the Compact Helical System Heliotron/Torsatron," Plasma Phys. Control. Fusion 42, A103 (2000).

[15] H. MAAßBERG et al., "The Neoclassical "Electron-Root" Feature in W7-AS," Proc. 24th EPS Conf. Contr. Fusion Plasma Phys., Berchtesgaden, 1997, Vol. 21A IV, p. 1605.

[16] J. BALDZUHN, M. KICK, H. MAAßBERG, and the W7-AS Team, "Measurement and Calculation of the Radial Electric Field in the Stellarator W7-AS," Plasma Phys Control. Fusion 40, 967 (1998).

[17] Y. TAKEIRI et al., "Formation of Electron Internal Transport Barrier and Achievement of High Ion Temperature in Large Helical Device," Phys. Plasmas 10, 1788 (2003).

[18] F. CASTEJON et al., "Enhanced Heat Confinement in the Flexible Heliac TJ-II,” Nucl. Fusion 42, 271 (2002). 
[19] K. IDA et al., "Characteristics of Transport in Electron Internal Transport Barriers and in the Vicinity of Rational Surfaces in the Large Helical Device,” Phys. Plasmas 11, 2551 (2004).

[20] F. CASTEJON et al., "Influence of Low-order Rational Magnetic Surfaces on Heat Transport in TJ-II Heliac ECRH Plasmas," Nucl. Fusion 44, 593 (2004).

[21] C.D. BEIDLER and W.N.G. HITCHON, "Ripple Transport in Helical-axis Advanced Stellarators: A Comparison with Classical Stellarator/Torsatrons," Plasma Phys. Control. Fusion 36, 317 (1994).

[22] H.E. MYNICK and W.N.G. HITCHON, "Effect of the Ambipolar Potential on Stellarator Confinement," Nucl. Fusion 23, 1053 (1983).

[23] W.I. van RIJ and S.P. HIRSHMAN, "Variational Bounds for Transport Coefficients in Three-dimensional Toroidal Plasmas," Phys. Fluids B 1, 563 (1989).

[24] V. TRIBALDOS, "Monte Carlo Estimation of Neoclassical Transport for the TJ-II Stellarator," Phys. Plasmas 8, 1229 (2001).

[25] S. MURAKAMI et al., "Neoclassical Transport Optimization of LHD,” Nucl. Fusion 42, L19 (2002).

[26] V.V. NEMOV, S.V. KASILOV, W. KERNBICHLER, and M.F. HEYN, "Evaluation of 1/v Neoclassical Transport in Stellarators," Phys. Plasmas 6, 4622 (1999).

[27] C.D. BEIDLER et al., "Neoclassical Transport in Stellarators - Results from an International Collaboration," 30th EPS Conf. Plasma Phys. Contr. Fus., St Petersburg 2003, http://epsppd.epfl.ch/StPetersburg/pdf/P3\_002.pdf.

[28] K. IDA et al., "Reduction of Ion Thermal Diffusivity Associated with the Transition of the Radial Electric Field in Neutral-Beam-Heated Plasmas in the Large Helical Device,” Phys. Rev. Lett. 86, 5297 (2001).

[29] S.K. CHAN, "Steady-state Kinetics of Diffusionless First Order Phase Transformations,” J. Chem. Phys. 67, 5755 (1977).

[30] H. MAAßBERG et al., "Experimental and Neoclassical Electron Heat Transport in the LMFP Regime for the Stellarators W7-A, L-2, and W7-AS," Phys. Fluids B 5, 3627 (1993).

[31] K. UO, “The Confinement of Plasma by the Heliotron Magnetic Field,” J. Phys. Soc. Japan 16, 1380 (1961).

[32] H.E. Mynick, T.K. Chu, and A.H. Boozer, “Class of Model Stellarator Fields with Enhanced Confinement,” Phys. Rev. Lett. 48, 322 (1982).

[33] M. YOKOYAMA and K.Y. WATANABE, "The Role of the Bumpy Field for the Ripple Diffusion in Strongly Inward Shifted Configurations in LHD," Nucl. Fusion 45, 1600 (2005).

[34] V. TRIBALDOS and B.PH. van MILLIGEN, "Electron Cyclotron Emission Calculations for TJ-II Stellarator," Nucl. Fusion 36, 283 (1996).

[35] V. TRIBALDOS et al., "Electron Cyclotron Heating and Current Drive in the TJ-II Stellarator," Plasma Phys. Control. Fusion 40, 2113 (1998).

[36] R.C. GOLDFINGER and D.B. BATCHELOR, "Theory of Electron Cyclotron Heating in the ATF Torsatron," Nucl. Fusion 27, 31 (1987).

[37] H. IDEI et al., "Formation of Positive Radial Electric Field by Electron Cyclotron Heating in Compact Helical System," Phys. Plasmas 1, 3400 (1994). 
[38] S. KUBO et al., "Extension and Characteristics of an ECRH Plasma in LHD," Plasma Phys. Control. Fusion 47, A81 (2005).

[39] E. WESTERHOF, "Wave Propagation through an Electron Cyclotron Resonance Layer," Plasma Phys. Contr. Fusion 39, 1015 (1997).

[40] M.A. BALAKINA, O.B. SMOLYAKOVA, and M.D. TOKMAN, "Numerical Simulations of Tangential Microwave Launching for EC Heating in a Tokamak," Plasma Physics Reports 29, 53 (2003).

[41] Y. TAKEIRI et al., "Electron ITB Formation with Combination of NBI and ECH in LHD," Fusion Sci. Techn. 46, 106 (2004).

[42] T. MINAMI et al., "Increased Understanding of Neoclassical Internal Transport Barriers in CHS," Nucl. Fusion 44, 342 (2004).

[43] U. GASPARINO et al., "Studies on Electron Cyclotron Heating at the Wendelstein VII-A/AS Stellarators," Plasma Phys. Control. Fusion 30, 283 (1988).

[44] V. TRIBALDOS and J. GUASP, "Neoclassical global flux simulations in stellarators," Plasma Phys. Control. Fusion 47, 545 (2005).

[45] M. ROMÉ et al., "Kinetic Modelling of the ECRH Power Deposition in W7-AS," Plasma Phys. Control. Fusion 39, 117 (1997).

[46] S. MURAKAMI et al., "5D Simulation Study of Suprathermal Electron Transport in Non-axisymmetric Plasmas," Proc. 17th IAEA Fusion Energy Conf., Yokohama, Japan, 1998, (IAEA, Vienna, 1999), Vol. 4, p. 1383.

[47] K. IDA et al., "Characteristics of Electron Heat Transport of Plasma with an Electron Internal-Transport Barrier in the Large Helical Device," Phys. Rev. Lett. 91, 085003 (2003).

[48] T. SHIMOZUMA et al., "Formation of Electron Internal Transport Barriers by Highly Localized Electron Cyclotron Resonance Heating in the Large Helical Device," Plasma Phys. Control. Fusion 45, 1183 (2003).

[49] H. MAAßBERG et al., "The neoclassical "Electron Root" Feature in the Wendelstein-7-AS Stellarator," Phys. Plasmas 7, 295 (2000).

[50] B. ZURRO et al., "Comparison of Impurity Poloidal Rotation, $T_{\mathrm{e}}$ and $T_{\mathrm{i}}$ in ECRH and NBI Discharges of the TJ-II Heliac," Fusion Sci. Techn., accepted for publication (2006).

[51] H. YAMADA et al., "Characterization of Energy Confinement in Net-current Free Plasmas using the Extended International Stellarator Database," Nucl. Fusion 45, 1684 (2005).

[52] M. ROMÉ et al., "The "Electron-root" Feature in the WENDELSTEIN-7-AS Stellarator with ECRH in O1-Mode Compared to X2-mode," Plasma Phys. Control. Fusion 48, 353 (2006).

[53] S. MURAKAMI et al., "5-D Simulation Study of Suprathermal Electron Transport in Non-axisymmetric Plasmas," Nucl. Fusion 40, 693 (2000).

[54] M. YOKOYAMA et al., "Towards Improved Confinement: Analysis of the Radial Electric Field in LHD," Nucl. Fusion 42, 143 (2002).

[55] S.V. KASILOV, W. KERNBICHLER, V.V. NEMOV, and M.F. HEYN, "Mapping Technique for Stellarators," Phys. Plasmas 9, 3508 (2002).

[56] S. MURAKAMI et al., "A Demonstration of Magnetic Field Optimization in LHD," Proc. 19th IAEA Fusion Energy Conf., Lyon, France, 2002 (IAEA, Vienna, 2002), paper IAEA-CN-94/EX-C5-3. 
[57] C.D. BEIDLER and W.D. D'HAESELEER, "A General Solution of the Ripple-averaged Kinetic Equation (GSRAKE),” Plasma Phys. Control. Fusion 37, 463 (1995).

[58] U. STROTH et al., "Internal Transport Barrier Triggered by Neoclassical Transport in W7-AS," Phys. Rev. Lett. 86, 5910 (2001).

[59] A. FUJISAWA et al., "Dynamic Behavior of Potential in the Plasma Core of the CHS Heliotron/Torsatron," Phys. Rev. Lett. 79, 1054 (1997).

[60] A. FUJISAWA et al., "Electric Pulsation and Profile Quantization in CHS Heliotron/Torsatron," Plasma Phys. Control. Fusion 41, A561 (1999).

[61] A. FUJISAWA et al., "Experimental Study of the Bifurcation Nature of the Electrostatic Potential of a Toroidal Helical Plasma," Phys. Plasmas 7, 4152 (2000).

[62] CH. FUCHS et al., "Shafranov Shift in the 'Electron Root' Feature at the W7-AS Stellarator," Fusion Eng. Design 53, 309 (2001).

[63] T. ESTRADA et al., "Electron Internal Transport Barriers and Magnetic Topology in the Stellarator TJ-II," Fusion Sci. Techn., accepted for publication (2006).

[64] N.J. LOPES CARDOZO et al., "Electron Thermal Transport in RTP: Filaments, Barriers and Bifurcations," Plasma Phys. Control. Fusion 39, B303 (1997).

[65] G. GORINI et al., “Transient Transport Barriers in Ohmic and Electron Cyclotron Heated RTP Plasmas: Relation with Rational Magnetic Surfaces," Plasma Phys. Control. Fusion 42, A161 (2000).

[66] S.V. KASILOV et al., "On the 'Magnetic' Nature of Electron Transport Barriers in Tokamaks," Plasma Phys. Control. Fusion 44, 985 (2002).

[67] H. WOBIG and D. PFIRSCH, "On guiding centre orbits of particles in toroidal systems," Plasma Phys. Control. Fusion 43, 695 (2001).

[68] K.C. SHAING et al., "Plasma and Momentum Transport Processes in the Vicinity of a Magnetic Island in a Tokamak," Nucl. Fusion 43, 258 (2003).

[69] V.N. KALYUZHNYJ and V.V. NEMOV, "Calculations of $1 / v$ Transport in an $l=3$ Stellarator Magnetic Field in the Presence of Magnetic Islands Caused by Magnetic System Errors," Fusion Sci. Techn. 46, 248 (2004). 


\section{Figure Captions}

Figure 1: $T_{\mathrm{e}}$-profiles for CERC discharges at (a) CHS ( $P_{\mathrm{ECH}} \approx 150 \mathrm{~kW}, n_{\mathrm{e}} \approx 0.3 \times 10^{19} \mathrm{~m}^{-3}$, $B=0.95 \mathrm{~T}),(\mathrm{b})$ LHD $\left(P_{\mathrm{ECH}} \approx 970 \mathrm{~kW}, n_{\mathrm{e}} \approx 0.15 \times 10^{19} \mathrm{~m}^{-3}, B=1.5 \mathrm{~T}\right),(\mathrm{c})$ TJ-II $\left(P_{\mathrm{ECH}}=200 \mathrm{~kW}\right.$, $\left.n_{\mathrm{e}} \approx 0.7 \times 10^{19} \mathrm{~m}^{-3}, B=0.95 \mathrm{~T}\right)$, and (d) W7-AS ( $\left.P_{\mathrm{ECH}} \approx 1.2 \mathrm{MW}, n_{\mathrm{e}} \approx 1.9 \times 10^{19} \mathrm{~m}^{-3}, B=2.5 \mathrm{~T}\right)$.

Figure 2: (a) $T_{\mathrm{e}}$-profiles and (b) $E_{\mathrm{r}}$-profiles measured by CXRS at LHD with $P_{\mathrm{ECH}}=0,0.58$ and $0.78 \mathrm{MW}$ on a NBI sustained target plasma [47]. CERC is established with $P_{\mathrm{ECH}} \geq 0.58 \mathrm{MW}$.

Figure 3: (a) The normalised scale-length of the $T_{\mathrm{e}}$-gradient as a function of $P_{\mathrm{ECH}} / n_{\mathrm{e}}$. (b) $T_{\mathrm{e}^{-}}$ profiles and (c) normalised electron heat diffusivities from the experimental power balance are shown for four different levels of $P_{\mathrm{ECH}} / n_{\mathrm{e}}[19]$.

Figure 4: A density scan with $P_{\mathrm{ECH}}=1.2 \mathrm{MW}$ in X2-mode at W7-AS for the "standard" configuration: (a) $n_{\mathrm{e}}$-profiles and (b) $T_{\mathrm{e}}$-profiles $\left(T_{\mathrm{e}}(0)\right.$ decreases with increasing $\left.n_{\mathrm{e}}\right)$.

Figure 5: Examples of measured $E_{\mathrm{r}}$ values compared with the neoclassical (NC) ambipolar $E_{\mathrm{r}}$ estimated from measured density and temperature profiles for CERC discharges at (a) CHS, (b) LHD, (c) TJ-II and (d) W7-AS. The "width" of NC ambipolar $E_{\mathrm{r}}$ in (c) is attributed to the uncertainty of profile measurements.

Figure 6: Comparison between the experimental (exp.) and neoclassical (NC) heat diffusivities, $\chi_{\mathrm{e}}(r)$, with and without considering the ambipolar $E_{\mathrm{r}}$ for (a) W7-AS [49] and (b) TJ-II [18].

Figure 7: Profiles of (a) the electrostatic potential measured by HIBP and (b) deduced $E_{\mathrm{r}}$ and (c) the density fluctuations (integrated from 5 to $70 \mathrm{kHz}$ ), before (dashed line) and after (solid line) CERC establishment at CHS. The thin solid line in (b) shows $d E_{\mathrm{r}} / d r$ for the case of CERC.

Figure 8: Time traces of the electrostatic potential, $\varphi, T_{\mathrm{e}}$ and the total beam intensity in the plasma core during transitions in TJ-II [10]. 
Common Features of Core Electron-Root Confinement in Helical Devices, M.Yokoyama et al.

Fig.1
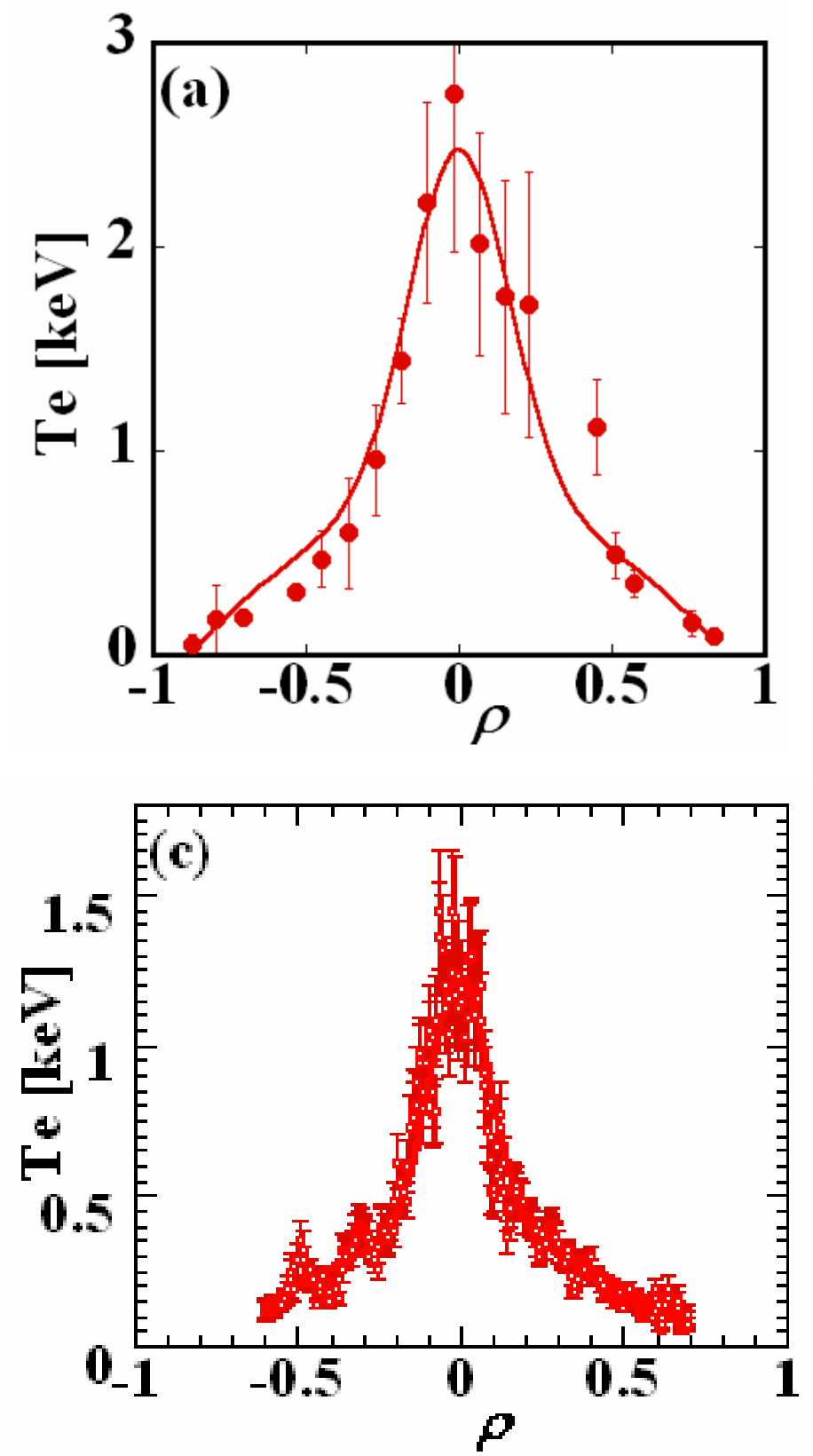
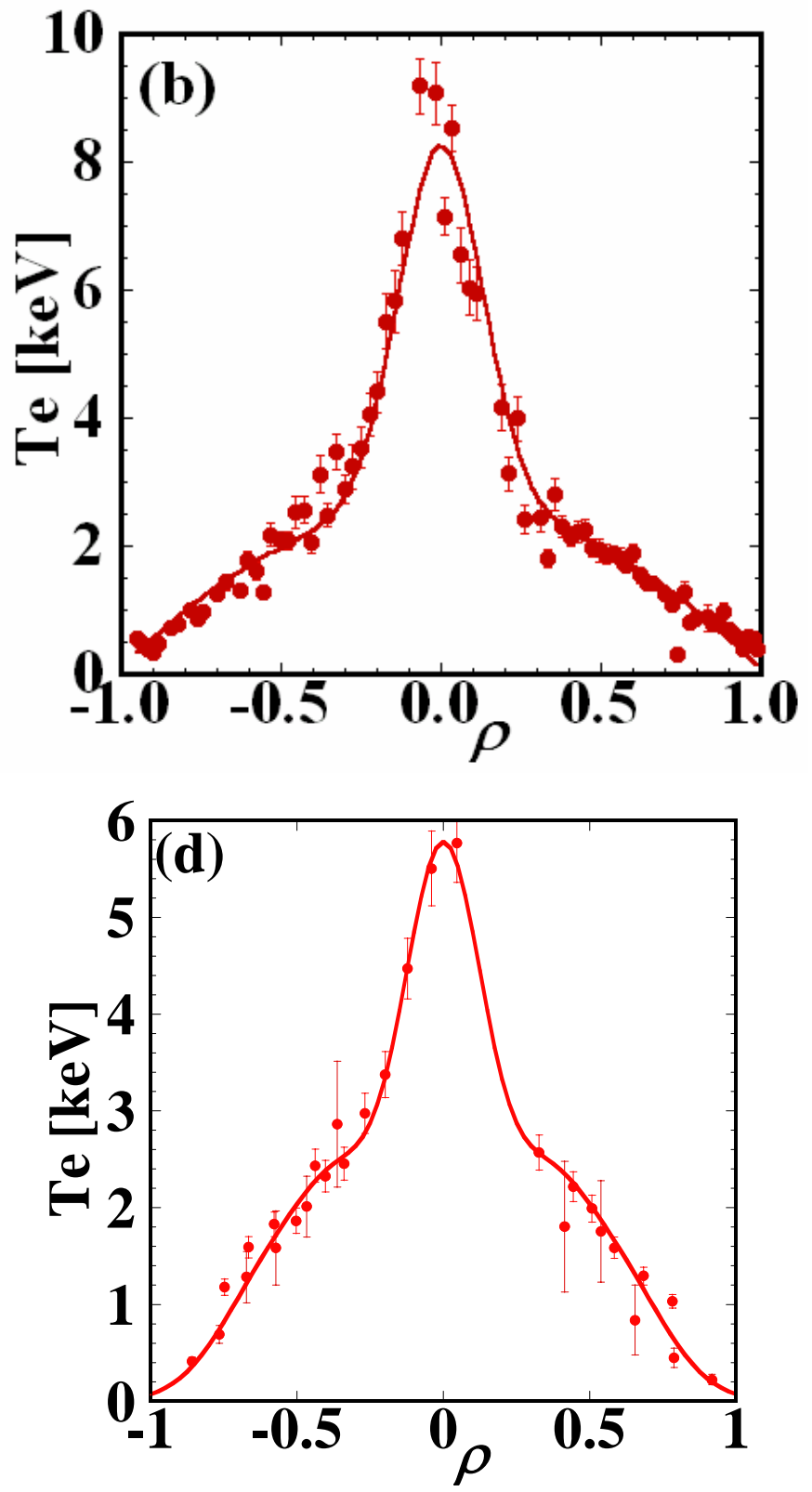

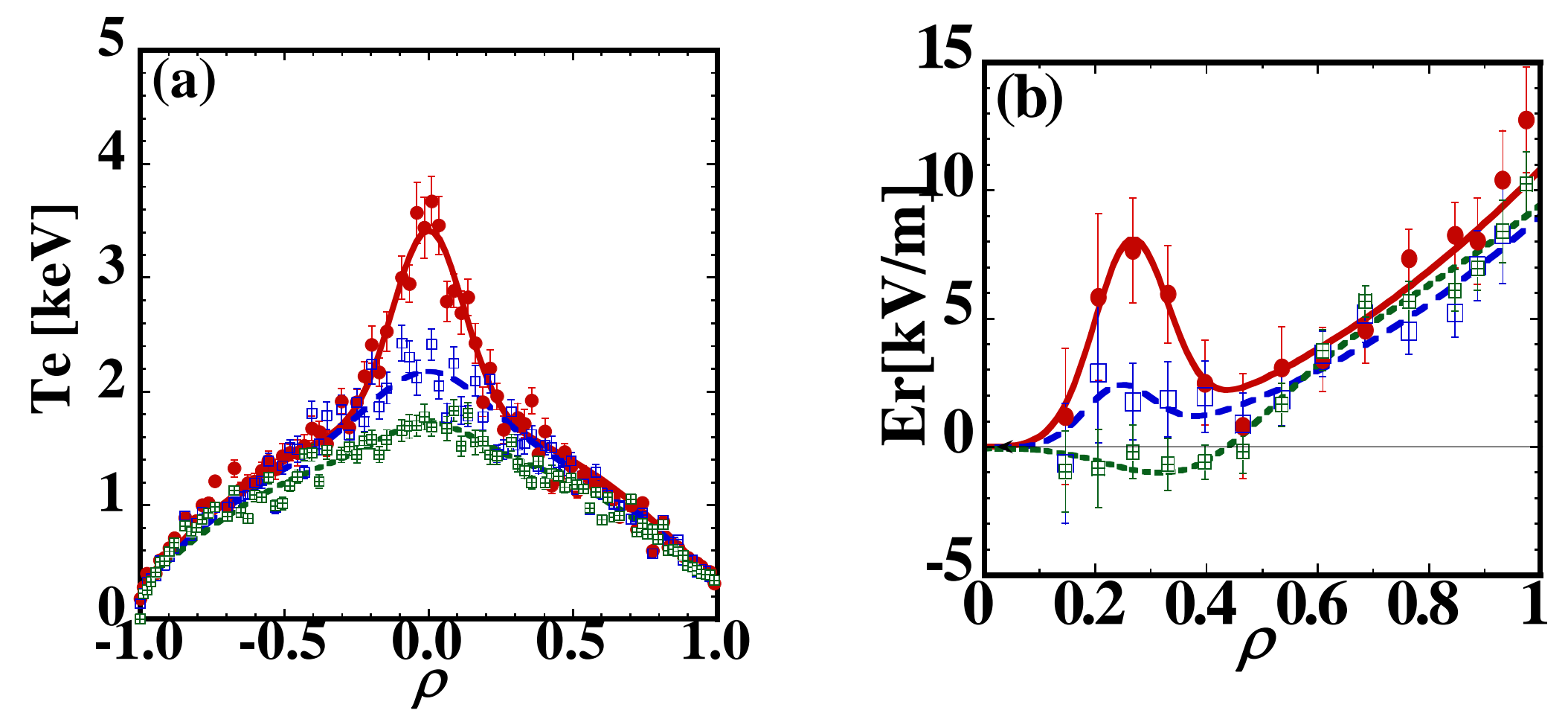
Common Features of Core Electron-Root Confinement in Helical Devices, M.Yokoyama et al.

Fig.3
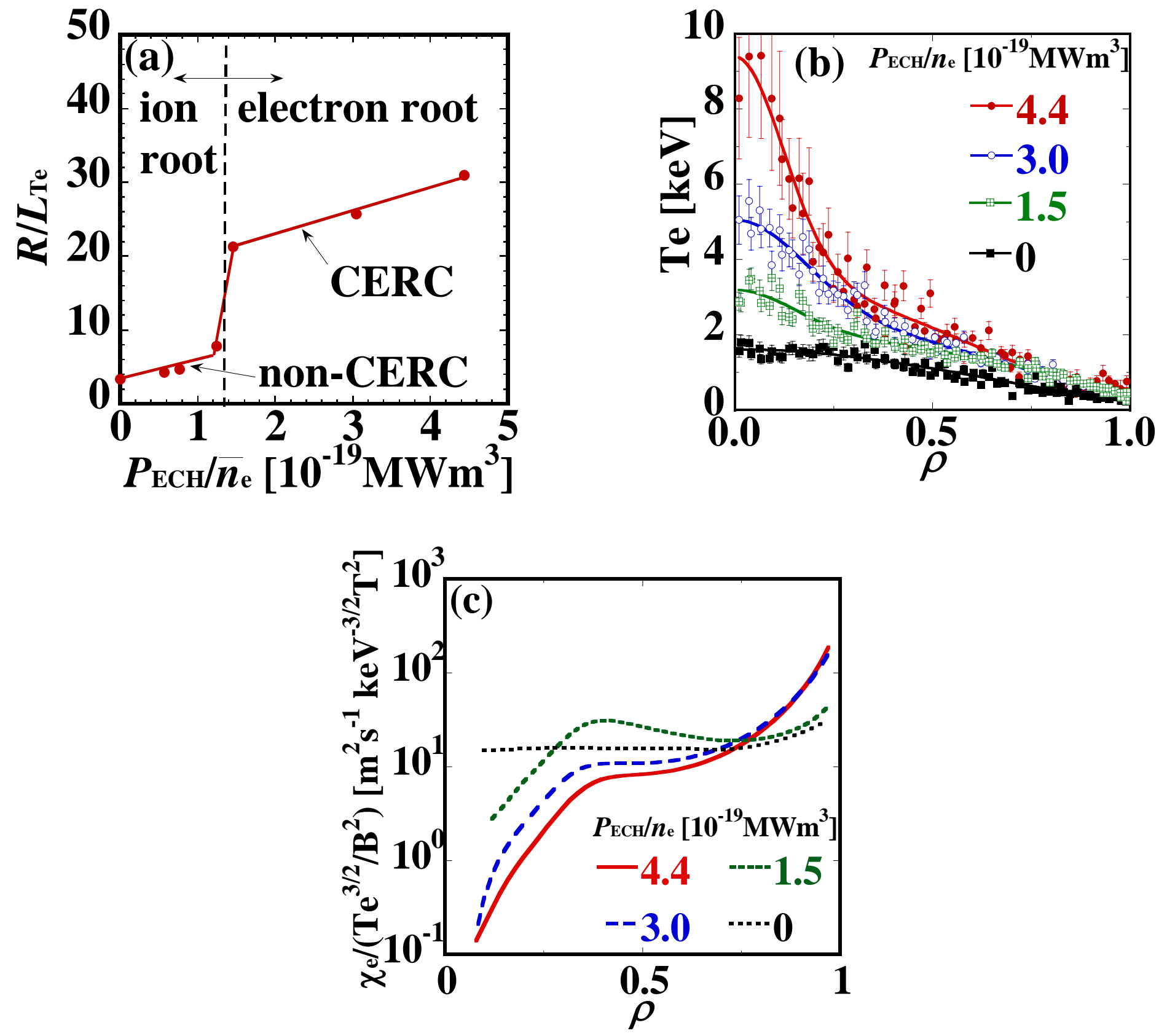
Common Features of Core Electron-Root Confinement in Helical Devices, M.Yokoyama et al.

Fig.4
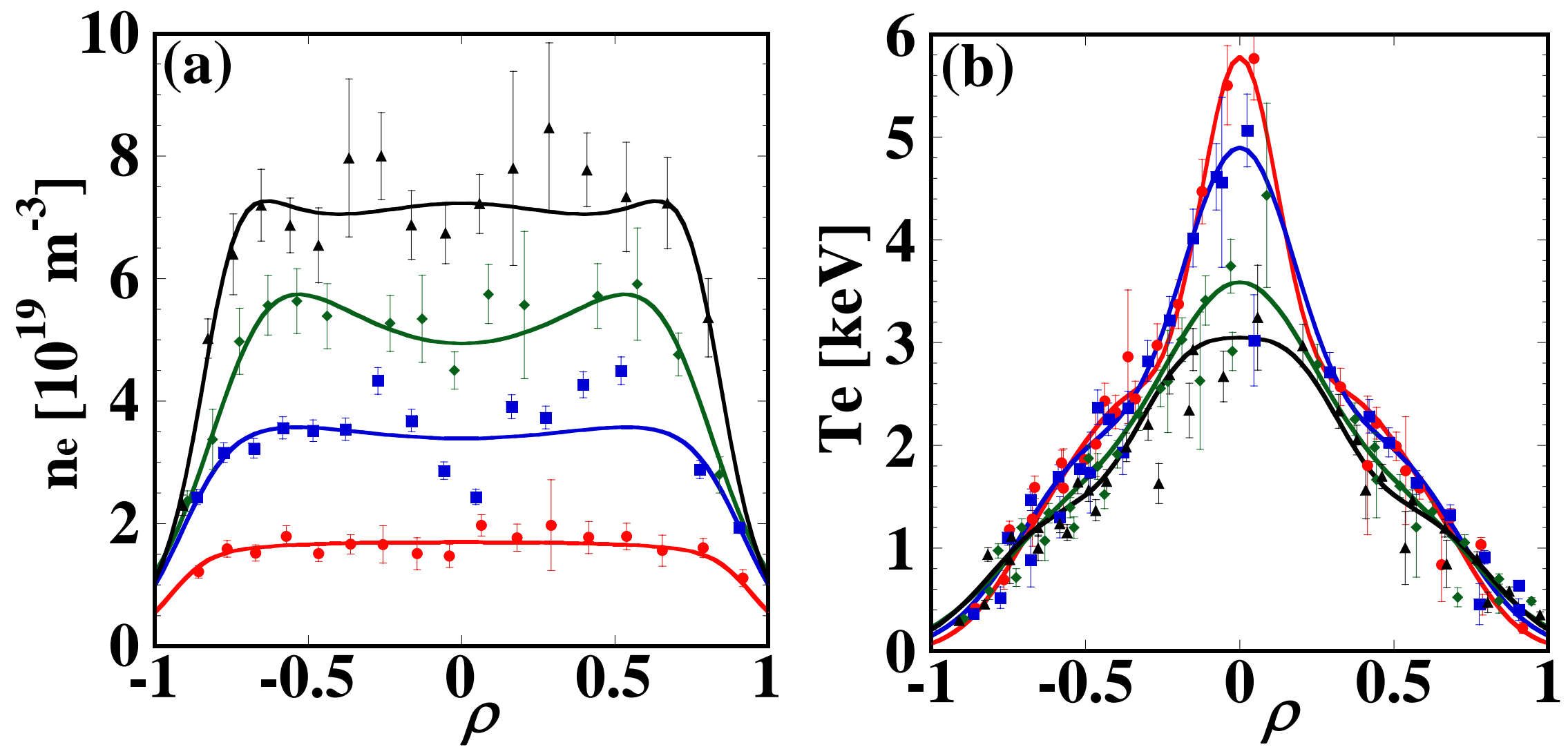
Fig.5

Common Features of Core Electron-Root Confinement in Helical Devices, M.Yokoyama et al.
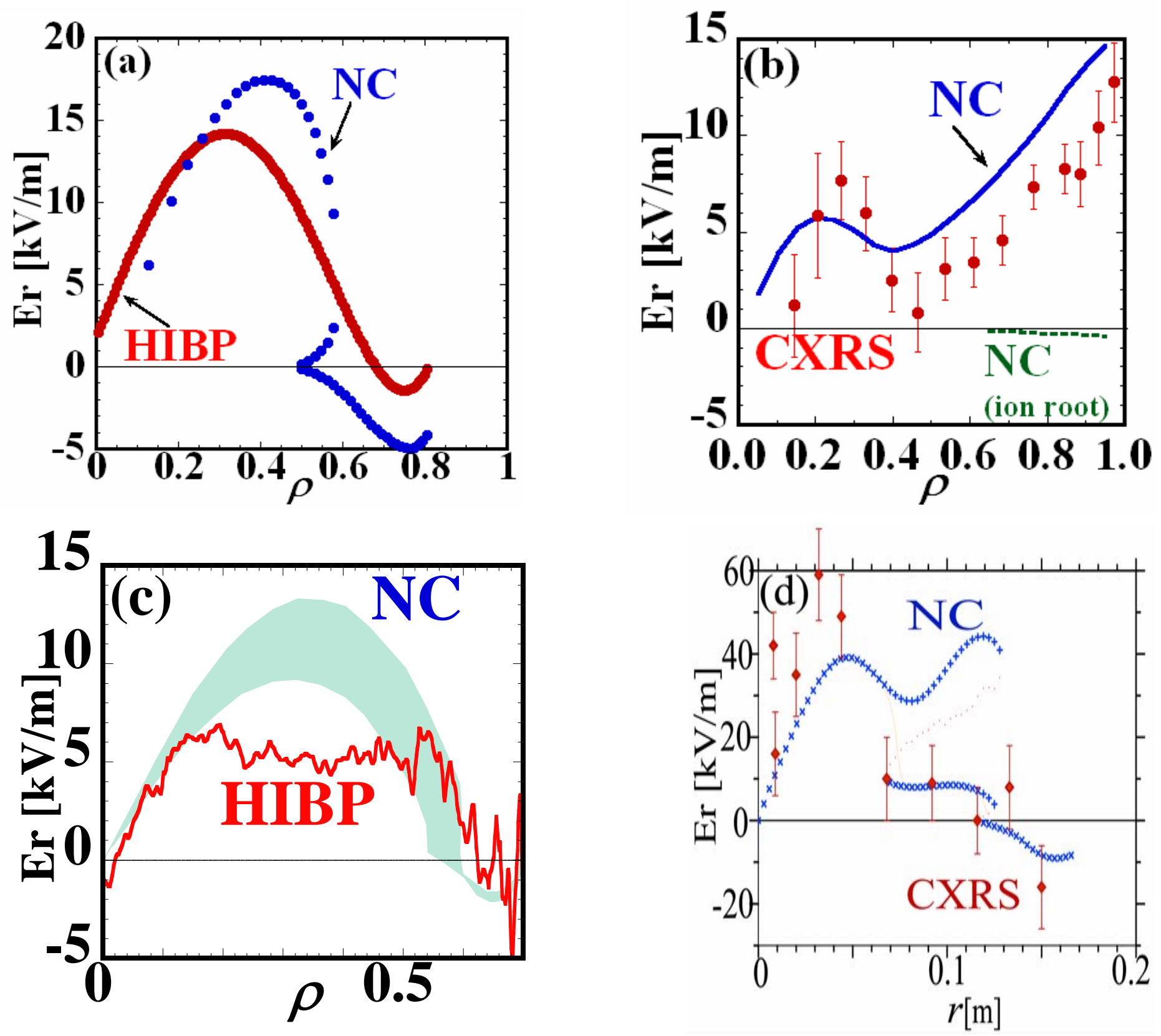

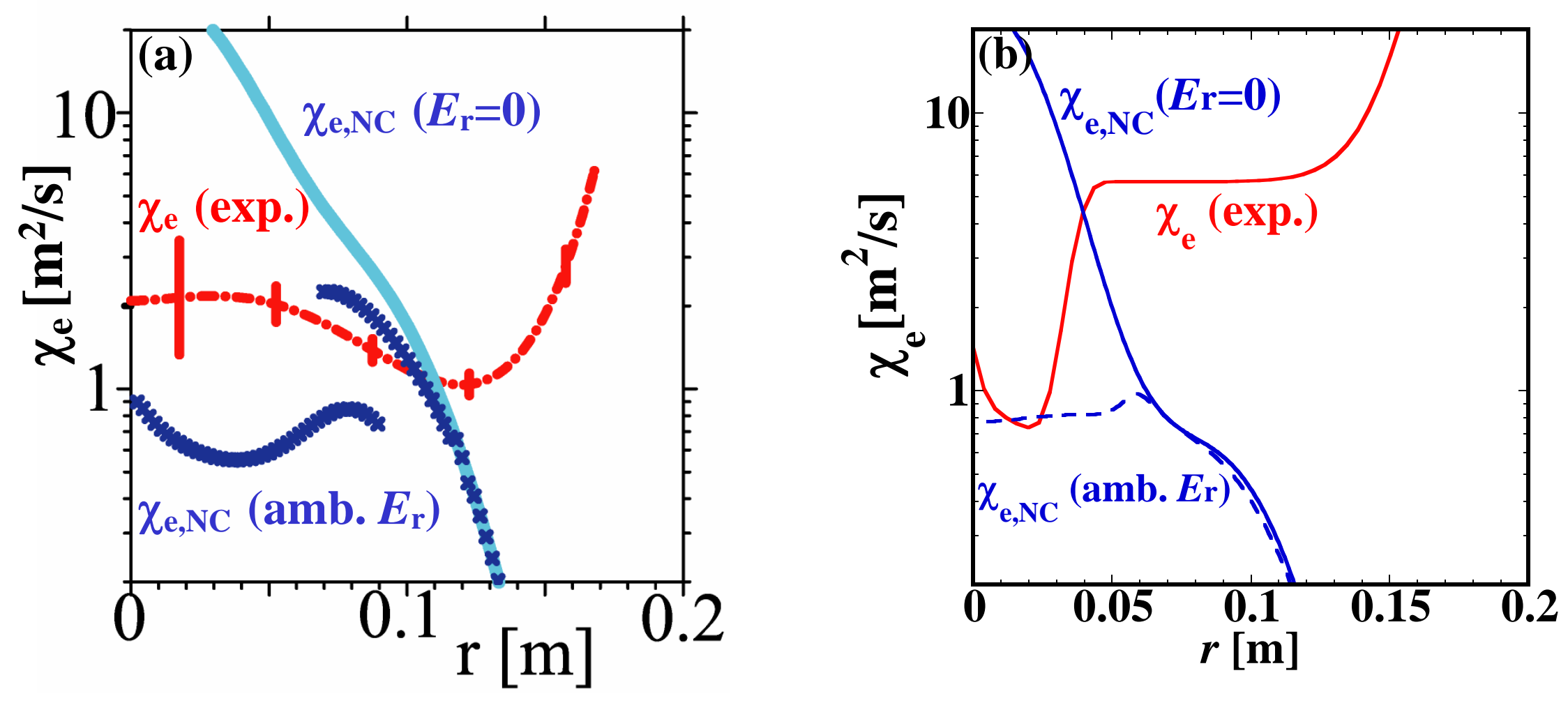

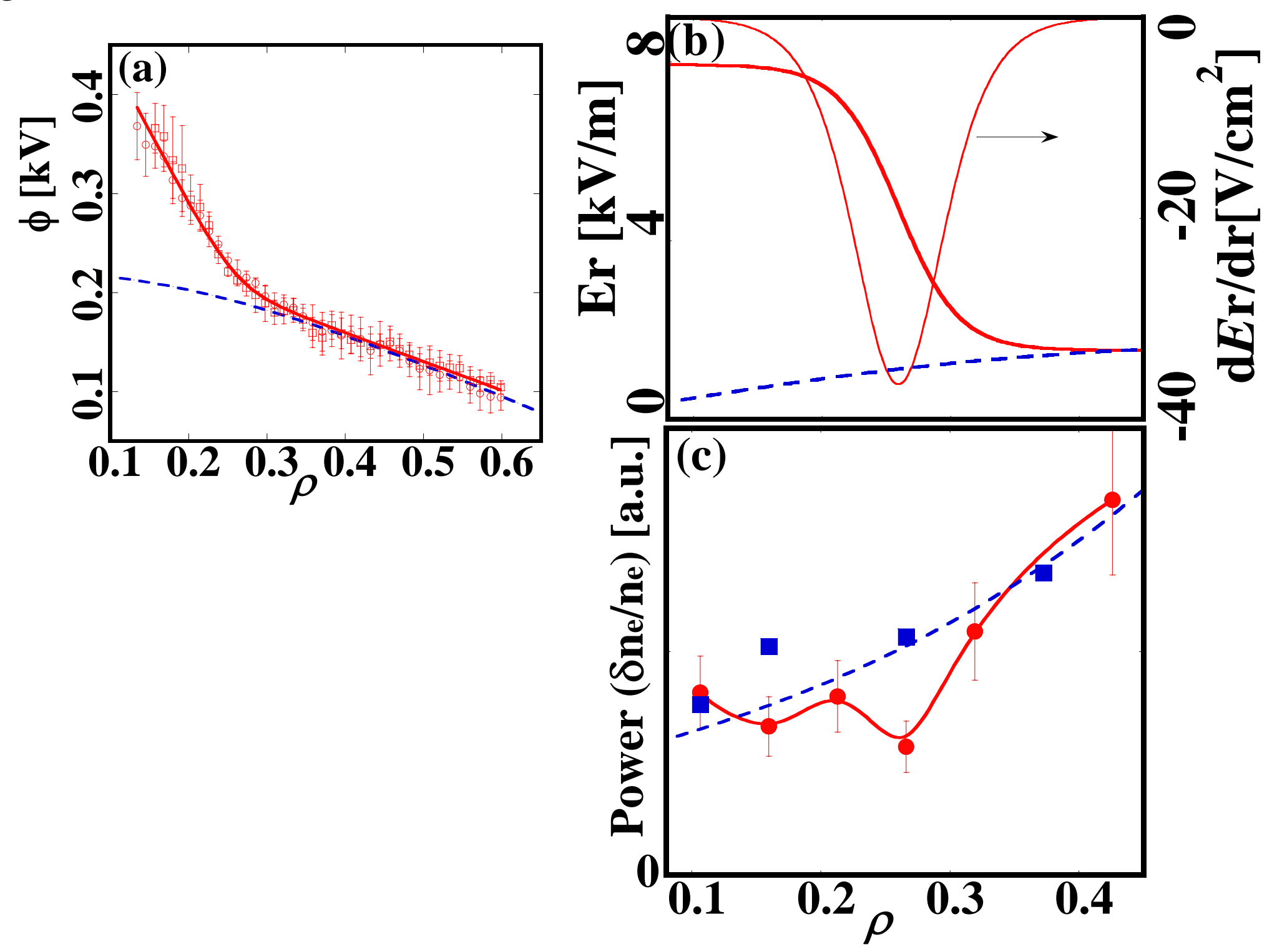

(b) and (c) have common X-axis 


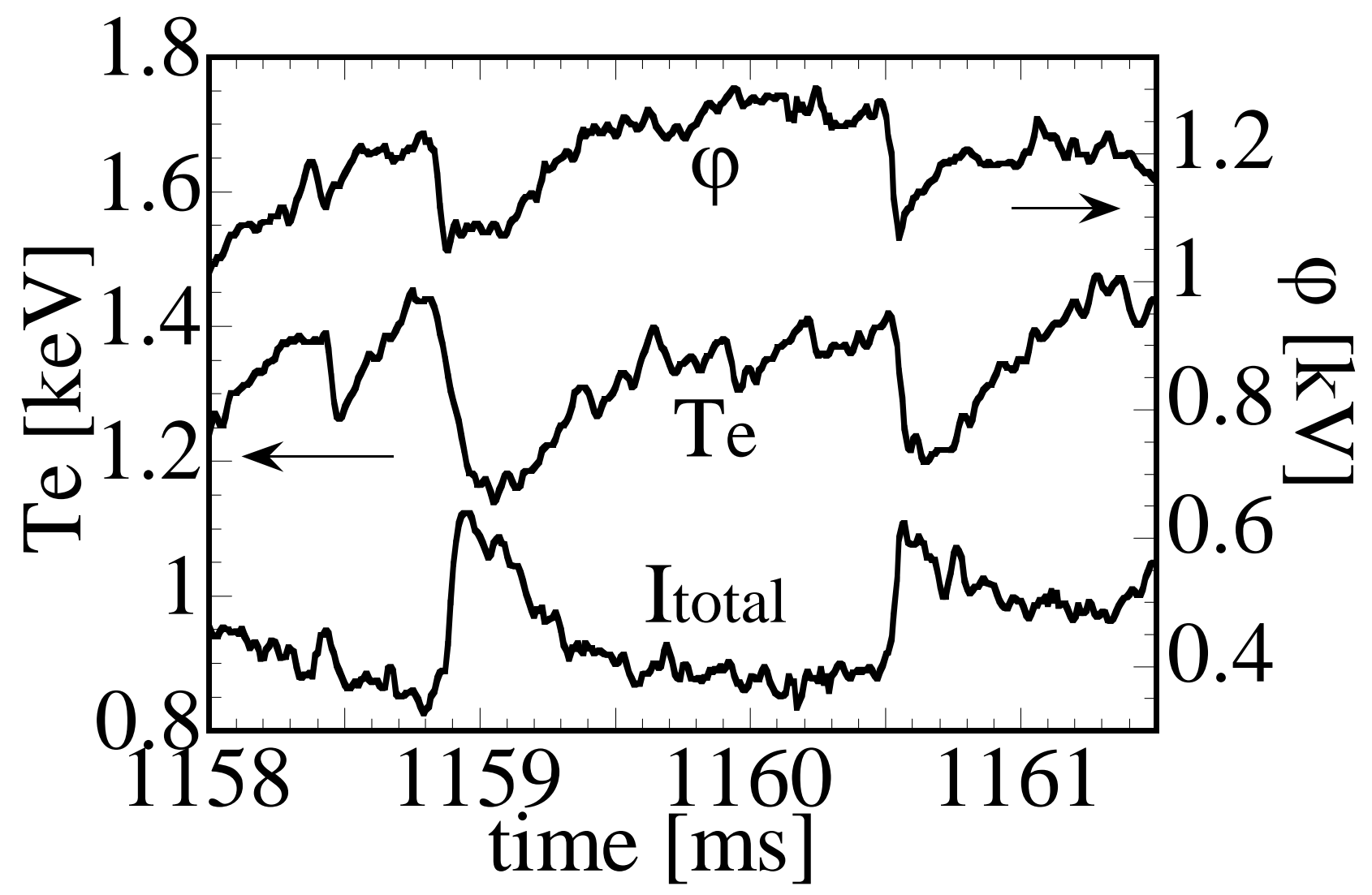

